ARTICLE

\title{
Indispensable epigenetic control of thymic epithelial cell development and function by polycomb repressive complex 2
}

Thomas Barthlott ${ }^{1,8}$, Adam E. Handel (10 2,8, Hong Ying Teh ${ }^{1,8}$, Rushika C. Wirasinha ${ }^{3}$, Katrin Hafen', Saulius Žuklys ${ }^{1}$, Benoit Roch ${ }^{4}$, Stuart H. Orkin (1) ${ }^{5}$, Jean-Pierre de Villartay (1) ${ }^{4}$, Stephen R. Daley ${ }^{3,7}$ \& Georg A. Holländer (i) ${ }^{1,6 \times}$

Thymic $T$ cell development and $T$ cell receptor repertoire selection are dependent on essential molecular cues provided by thymic epithelial cells (TEC). TEC development and function are regulated by their epigenetic landscape, in which the repressive H3K27me3 epigenetic marks are catalyzed by polycomb repressive complex 2 (PRC2). Here we show that a TEC-targeted deficiency of PRC2 function results in a hypoplastic thymus with reduced ability to express antigens and select a normal repertoire of $T$ cells. The absence of PRC2 activity reveals a transcriptomically distinct medullary TEC lineage that incompletely off-sets the shortage of canonically-derived medullary TEC whereas cortical TEC numbers remain unchanged. This alternative TEC development is associated with the generation of reduced TCR diversity. Hence, normal PRC2 activity and placement of H3K27me3 marks are required for TEC lineage differentiation and function and, in their absence, the thymus is unable to compensate for the loss of a normal TEC scaffold.

\footnotetext{
${ }^{1}$ Department of Biomedicine and University Children's Hospital of Basel, University of Basel, Basel, Switzerland. ${ }^{2}$ Nuffield Department of Clinical Neurosciences, University of Oxford, Oxford, UK. ${ }^{3}$ Infection and Immunity Program, Monash Biomedicine Discovery Institute and Department of Biochemistry and Molecular Biology, Monash University, Melbourne, VIC, Australia. ${ }^{4}$ Genome Dynamics in the Immune System Laboratory, INSERM UMR 1163, Université de Paris, Imagine Institute, Paris, France. ${ }^{5}$ Department of Pediatric Oncology, Dana-Farber Cancer Institute and Division of Hematology/ Oncology, Boston Children's Hospital, Harvard Stem Cell Institute, Harvard Medical School, and Howard Hughes Medical Institute, Boston, MA, USA. ${ }^{6}$ Department of Paediatrics and the Weatherall Institute of Molecular Medicine, University of Oxford, Oxford, UK. 'Present address: School of Health and Biomedical Sciences, Queensland University of Technology, Brisbane, QLD, Australia. ${ }^{8}$ These authors contributed equally: Thomas Barthlott, Adam E. Handel, Hong Ying Teh. ${ }^{{ }_{e}}$ email: georg.hollander@paediatrics.ox.ac.uk
} 
$\mathrm{T}$ he controlled balance between cell proliferation and differentiation is essential to maintain a normal thymic microenvironment to promote the development and selection of naive $\mathrm{T}$ cells with a repertoire purged of vital "self" specificities but prepared to react to injurious "non-self". Thymic epithelial cells (TECs), which can be categorized based on specific molecular, structural and functional characteristics into separate cortical (cTECs) and medullary (mTECs) lineages, are essential for this competence ${ }^{1}$. Both TEC lineages derive from a common epithelial precursor of endodermal origin ${ }^{2,3}$ that expresses a range of cTEC-specific markers including the proteasome component $\beta 5$ t encoded by Psmb11 ${ }^{4-7}$.

cTECs induce the commitment of blood-borne precursor cells to a $\mathrm{T}$ cell fate, foster their subsequent growth and initial maturation, and select immature thymocytes that express a $\mathrm{T}$ cell antigen receptor (TCR) with sufficient affinity for self-peptides presented by major histocompatibility complexes (pMHC). In contrast, mTECs promote the terminal differentiation of thymocytes, which includes the establishment of immunological tolerance to tissue-restricted antigens (TRAs) via two complementary mechanisms; a recessive activity of deleting thymocytes expressing a TCR with high affinity for these antigens, and a dominant mechanism of clonal diversion that generates thymic regulatory $\mathrm{T}$ cells ${ }^{8}$. In addition, thymus immigrating dendritic cells (DCs) and B cells provide peripheral antigens for central tolerance induction ${ }^{9-11}$.

The ectopic representation of a significant number of TRAs by a subpopulation of phenotypically mature mTECs is in part dependent on the expression of the transcriptional facilitator autoimmune regulator (AIRE) $)^{12}$, which acts independently of lineage-specific transcription factors ${ }^{13}$. Individual mTECs express AIRE-dependent TRAs to an extent significantly lower than that of corresponding peripheral tissues ${ }^{14,15}$ and in co-expression patterns that change with mTEC maturation ${ }^{16-19}$. AIRE limits the amplitude of TRA gene expression by repressing chromatin accessibility at distal cisregulatory sequences ${ }^{20}$. These regions, ostensibly placed within facultative heterochromatin, are enriched in the repressive histone mark, H3K27me3, and depleted for histone modifications permissive for conventional gene transcription ${ }^{8}$.

H3K27me3 is a posttranslational modification catalysed by polycomb repressive complex 2 (PRC2) that depends for its function on four core factors, including EED (embryonic ectoderm development), which is critical for physically binding to H3K27me3, and both EZH1 and EZH2 (enhancer of zeste homologue), which mediate the trimethylation of $\mathrm{H} 3 \mathrm{~K} 27^{21,22}$. Compromised gene repression due to a loss of PRC2 activity ${ }^{23}$ impairs tissue specification and maintenance, and consequently results in early embryonic lethality ${ }^{24}$.

To establish a specific role for PRC2 in TEC development and function, we analyse mice with a loss of this complex targeted to TEC precursors and occurring after the formation of the thymus anlage. Absent PRC2 activity in TEC leads to an impairment in canonical TEC development and function whilst in parallel an alternative mTEC differentiation pathway emerges. These abnormalities are associated with reduced TCR diversity in the $\mathrm{CD}^{+}$and $\mathrm{CD} 8^{+}$naive $\mathrm{T}$ cell pools, yet negative selection of selfreactive TCR specificities remained intact. Taken together, we conclude that PRC2 activity is essential for TEC lineage specification and function.

\section{Results}

Loss of EED in TEC differentially disrupts lineage development. To generate mice with a loss of PRC2 activity in all thymic epithelia at a time after initiation of a thymus anlage, we interbred animals harbouring a conditional Eed allele to mice in which the
Cre recombinase is expressed under the control of Psmb11 reg-

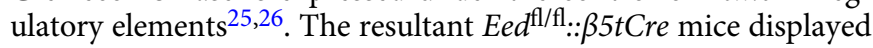
a small thymus with a reduced total cellularity, a normal corticomedullary segregation and a number of small, cell-free cysts surrounded by cytokeratin 8-positive epithelia (Fig. 1a-d). However, the total number of TEC recovered from these mice was similar to that of wild-type and Eed $d^{\mathrm{f} / \mathrm{fl}}$ mice (Fig. 1e). EED deletion resulted in the expected depletion of $\mathrm{H} 3 \mathrm{~K} 27$ me3 marks in all cTECs and the majority of mTECs isolated from $E e d^{\mathrm{fl} / \mathrm{fl}: \text { : }}$ $\beta 5 t$ Cre mice, whereas $\mathrm{H} 3$ histone abundance remained unchanged (Fig. If and see below; gating strategy in Supplementary Fig. 1).

$E d^{\mathrm{f} / \mathrm{fl}}:: \beta 5 t$ Cre mice had a higher frequency and cellularity of cTECs but a lower rate and reduced cell number of mTECs (Fig. 2a). The difference was present at birth and steadily increased with age. Noticeably, cTEC cellularity did not diminish in adolescent Eed ${ }^{\mathrm{f} / \mathrm{fl}}:: \beta 5 t \mathrm{Cre}$ mice contrary to controls. Moreover, the maturation of mTEC was compromised in Eed: $\mathrm{f}^{\mathrm{l} / \mathrm{fl}}: \beta 5 t \mathrm{tre}$ mice with fewer immature epithelia $\left(\mathrm{MHCII}^{\mathrm{lo}}\right.$, $\mathrm{AIRE}^{-}$, designated mTEC $^{l o}$ ) attaining a mature (MHChi; mTEC $^{\text {hi }}$ ) cell stage, either positive or negative for the expression of AIRE (Fig. 2b). Heterozygosity for a loss of Eed did not compromise thymus cellularity nor affect TEC numbers or subset composition (Fig. 2c). The proliferation rates of both CTEC and mTEC were significantly reduced in Eed ${ }^{\mathrm{f} / \mathrm{fl}}:: \beta 5 t \mathrm{Cre}$ mice (Fig. $1 \mathrm{~h}$ and Supplementary Fig. 1) rendering it an unlikely explanation for the disparity in CTEC:mTEC ratios. Thus, the loss of PRC2 activity (consequent to an absence of either EED or EZH1/2; Supplementary Fig. 2) resulted in an expansion of cTECs but a reduction in mTECs. One possible explanation for this finding could be that TEC precursors are preferentially differentiating into the cTEC lineage.

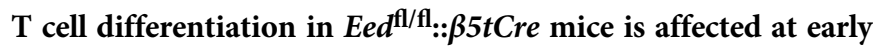
and late stages. We next evaluated the thymopoiesis of 3-week-

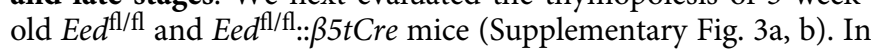
the absence of EED, the proportion of cells in the $\mathrm{CD} 4^{-} \mathrm{CD} 8^{-}$ double-negative (DN) thymoycte compartment increased 3-4fold $(2.5 \pm 0.4 \%$ vs. $8.1 \pm 0.9 \%$; Fig. $3 \mathrm{a})$. This relative increase was mainly due to the presence of $\mathrm{B}$ cells although their absolute numbers were not altered $\left(4.8 \pm 0.6 \times 10^{5}\right.$ in Eed ${ }^{\mathrm{fl} / \mathrm{fl}:: \beta 5 t C r e}$ and $3.2 \pm 1.3 \times 10^{5}$ in $\left.E e d^{\mathrm{fl} / \mathrm{fl}}\right)$. The relative number of early thymic progenitors (ETP, defined as DN CD44 ${ }^{\mathrm{hi}} \mathrm{ckit}^{\mathrm{hi}} \mathrm{Sca}-\mathrm{1}^{\text {pos }} \mathrm{CD} 25^{-}$) and DN2 (DN CD44hickit ${ }^{\text {hisca-1 }}{ }^{\text {pos }} \mathrm{CD} 25^{+}$) cells in relation to all thymocytes of the $\alpha \beta \mathrm{T}$ cell lineage (i.e. excluding cells positive for TCR $\gamma \delta$, CD19, CD11b, CD11c, DX5, NK1.1, Gr1, F4/80, Ter119 and MHCII) was comparable in mutant and wild-type mice (Fig. 3b and Supplementary Fig. 3a). We observed, however, a partial block at the DN3 (i.e. DN CD $44^{-} \mathrm{CD} 25^{+}$) $\beta$-selection checkpoint. Compared to DN2 cells, DN3 thymocytes exhibited an almost 2-fold reduction in CD25 expression, which was paralleled by a significant reduction in the frequency of $\mathrm{CD} 71^{+}$predouble-positive (preDP) thymocytes in the ckit ${ }^{\text {neg }}$ DN subpopulation $(27.5 \pm 2.1 \%$ vs. $38.7 \pm 2.8)$. The progression through subsequent maturation stages (i.e. $\mathrm{CD} 71^{+} \mathrm{CD}^{+}$immature singlepositive (ISP) and $C D 71^{+} \mathrm{DP}$ ) was, however, not affected in mutant mice (Fig. 3c). Hence, these results indicate a partial block at the $\beta$-selection checkpoint caused by an EED deficiency in TEC. Whether the reduction in CD25 expression in DN3 is causally linked to the observed decrease in the generation of preDP thymocytes remains to be determined.

We next analysed positive selection into the single-positive (SP) thymocytes. Although the frequencies of positively selected DPdull cells were unchanged, we detected in Eed:fl/fl: $\beta 5 t$ Cre mice an accumulation of both CD8SP and CD4SP ( $\mathrm{T}_{\text {reg }}$ excluded) 
a

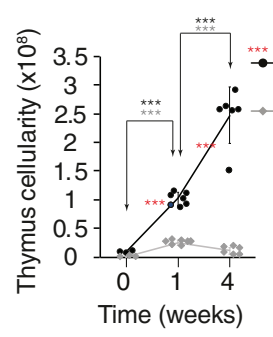

b Eeditut Eed ${ }^{\text {d/ft/l:: }}$ $\beta 5 t C r e$ d

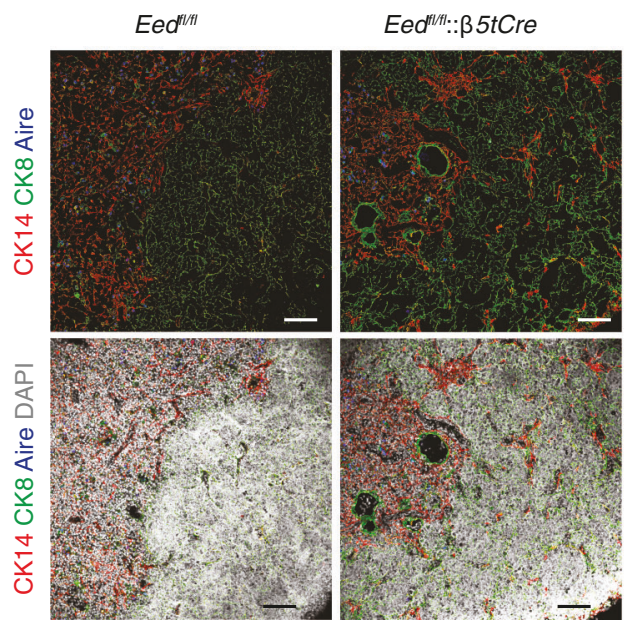

C

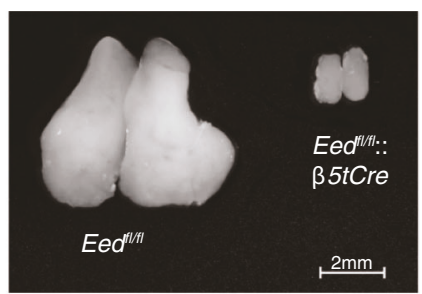

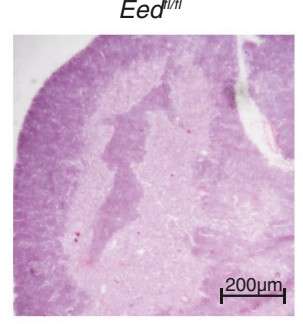

Eed $^{[t / t !::}: \beta 5 t C r e$

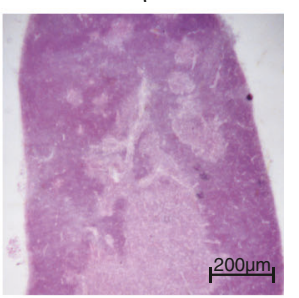

e

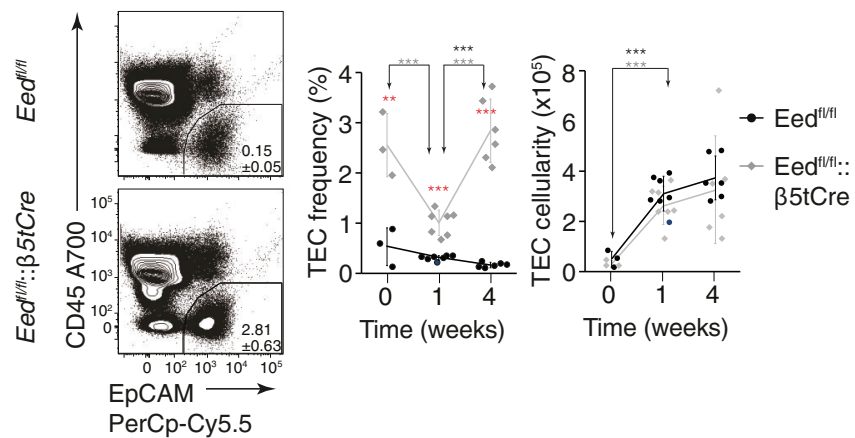

$\mathbf{f}$

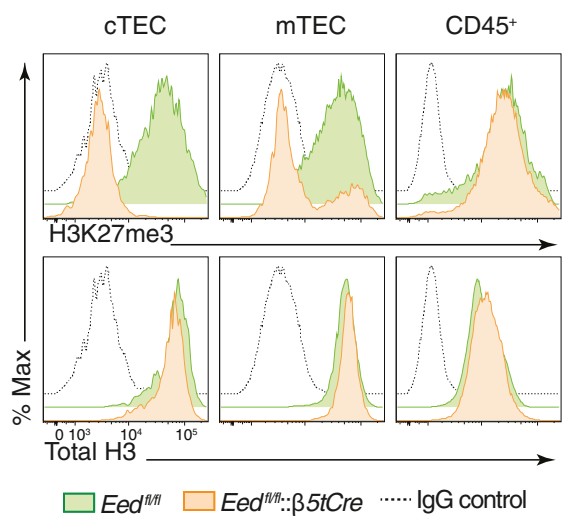

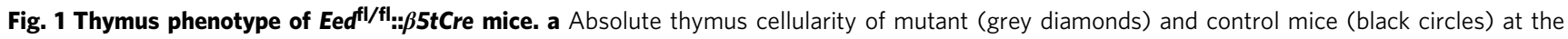
indicated postnatal ages (week 0 relates to newborn). b Macroscopic and c microscopic comparison of thymic lobes isolated from 4-week-old Eed ${ }^{f l / f l}:$ : $\beta 5 t C r e$ and control Eed ${ }^{f l / f l}$ mice. Scale bars represent $2 \mathrm{~mm}$ and $200 \mu \mathrm{m}$, respectively. Tissue sections were stained with haematoxylin and eosin. $\mathbf{d}$

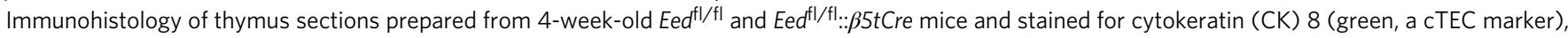
CK14 (red, an mTEC marker), AIRE (blue) and DAPI (grey; only in lower panels). Scale bars represent $50 \mu$ m. e Relative frequency and absolute cellularity of TEC isolated from Eed ${ }^{f l / f l}:: \beta 5 t C r e$ (grey diamonds) and control Eed ${ }^{f l / f l}$ mice (black circles) at indicated ages. Contour plots show representative flow cytometric analyses of thymic single-cell suspensions of 4-week-old mice, the gating for TEC and their relative frequencies. $\mathbf{f}$ Detection of H3K27me3

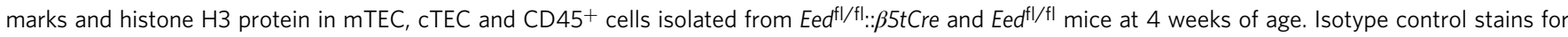
Eed ${ }^{f / f \mid}$ mice are indicated as $\operatorname{lgG}$ control. Data in graphs $(\mathbf{a}, \mathbf{e})$ represent mean and standard deviations (SD) and are pooled from independent experiments: week 0: $n=3$, week 1: $n=7$, week 4: $n=6 ;{ }^{*} p<0.05,{ }^{* *} p<0.01,{ }^{* \star *} p<0.001$ (two-tailed unpaired Student's $t$ test). Asterisks in red indicate comparisons between $E e d^{f \mid / f l}:: \beta 5 t C r e$ and Eed ${ }^{f l / f l}$ mice, whereas black and grey asterisks compare results from identical mouse strains at different time points. Data in (bd, f) are representative of two independent experiments with $n=3$ mice each per strain investigated. $n=$ biologically independent replicates per group. The gating for thymic epithelia is displayed in Supplementary Fig. 1. Source data including exact statistical test values are provided in the Source Data file. 
a

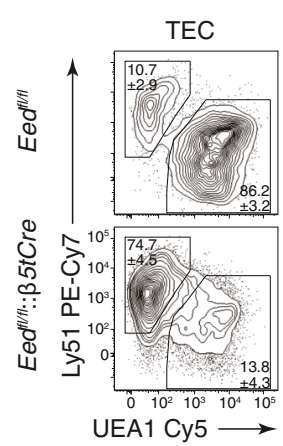

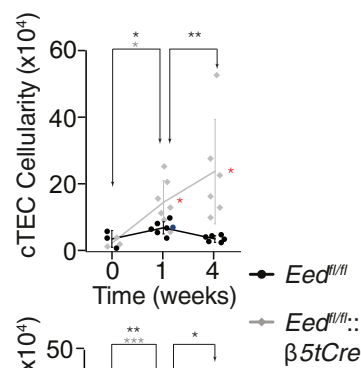

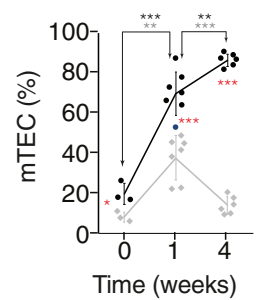

b

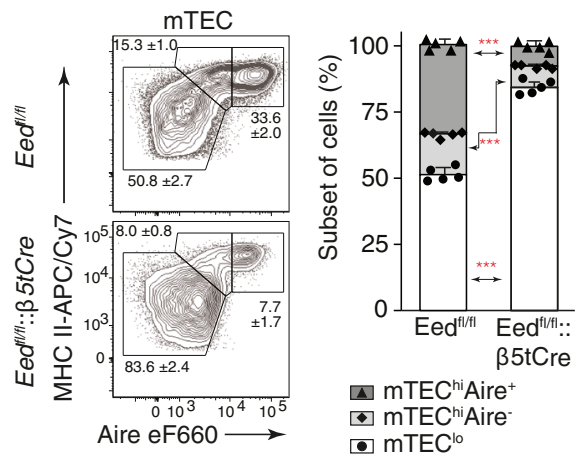

C
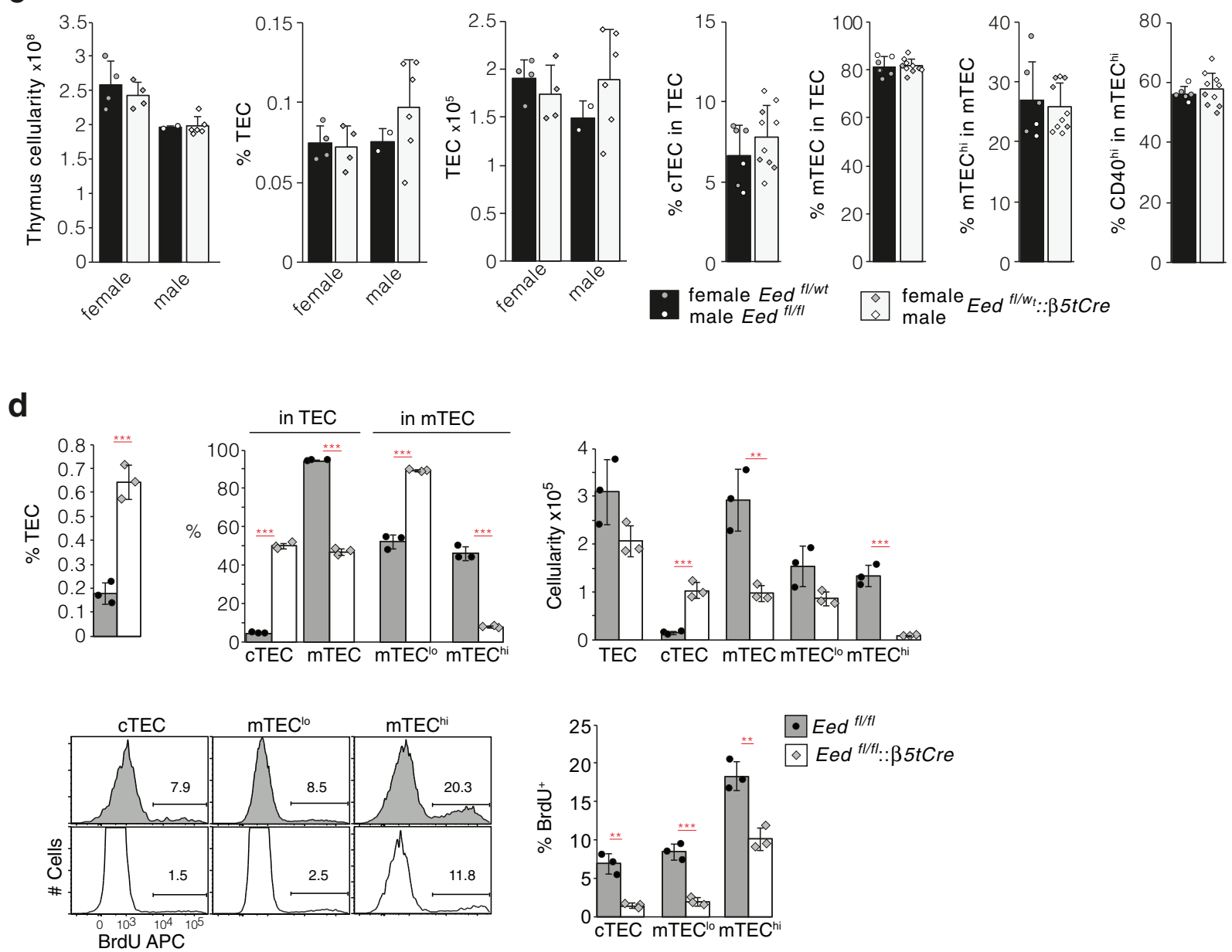

mature cells with a $\mathrm{CD}^{-} 9^{-} \mathrm{CD} 24^{-}$phenotype (CD8SP: $56.5 \pm$ $2.5 \%$ vs. $41.8 \pm 2.6 \%$; CD 4 SP: $45.4 \pm 4.3 \%$ vs. $28.5 \pm 0.5 \%$, Fig. $3 \mathrm{~d}$, e). Both lineages displayed a normal progression from DPdull to $\mathrm{CD} 9^{+} \mathrm{CD} 24^{+}$immature SP stages, suggesting that the relative increase of mature $\mathrm{CD} 24^{-} \mathrm{SP}$ was caused by retention in the thymus and not by an altered developmental transition. Although the overall frequency of Foxp $3^{+} \mathrm{T}_{\text {reg }}$ cells within CD4 SP thymocytes was only marginally, yet significantly elevated in mutant mice, their phenotype was severely skewed towards a CD24 ${ }^{-}$CCR7 $7^{\text {lo }}$ expression pattern $(67.4 \pm 5.0 \%$ vs. $28.9 \pm 3.2 \%)$ indicative of extended residence of cells in the medulla and/or re- circulation from the periphery back to the thymus (Fig. 3e). Within that $\mathrm{CD}_{24}^{-}$subset, the frequency of re-circulated peripheral T cells with a CD $44^{+}$phenotype (excluded from the

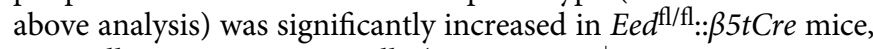
especially among CD8SP cells $\left(\mathrm{CD} 24^{-} \mathrm{CD} 8 \mathrm{:}^{+} 16.4 \pm 5.3 \%\right.$ vs. 3.5 $\pm 0.8 \%$; $\mathrm{CD}_{2} 4^{-} \mathrm{CD} 4^{+}: 2.7 \pm 0.5 \%$ vs. $1.2 \pm 0.2 \%$, Fig. $3 \mathrm{f}$ and Supplementary Fig. 3b). Hence, the absence of PRC2 function in TEC caused an accumulation of specific SP thymocytes.

Next, we characterized the negative selection of CD4 lineage thymocytes. The first wave of negative selection within the cortex (as identified by the co-expression of Helios and PD1 on Foxp3- 


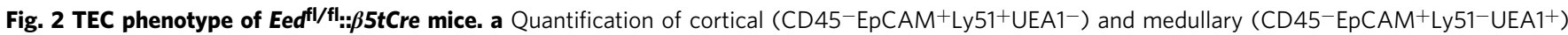
TEC subpopulations isolated from mice with the indicated genotype. Representative contour plots are from 4-week-old mice, frequencies and absolute cell numbers of cTEC and mTEC are from mice with the indicated genotype (Eed ${ }^{f l / f l}: . \beta 5 t C r e:$ grey diamonds, Eed ${ }^{f l / f l}$ mice: black circles) at ages shown (week 0 : $n=3$, week 1: $n=7$, week 4: $n=6$ ). b Flow cytometric analysis of mTEC from 4-week-old mice for MHCll and AIRE expression. Representative contour plots and bar graphs displaying the frequencies of individual subpopulations (mTEC:lo white bars black circles, mTEChi AIRE:- light grey bars black diamonds, mTEChi AIRE:+ grey bars black triangles) for each of the indicated genotypes $(n=5)$. c Quantification of total and TEC cellularity and determination of TEC subset distributions in Eed ${ }^{\mathrm{f} / \mathrm{wt} .: .} \beta 5 \mathrm{tCre}$ and control mice. Data are pooled from two independent experiments with female $(n=4$ Eed:

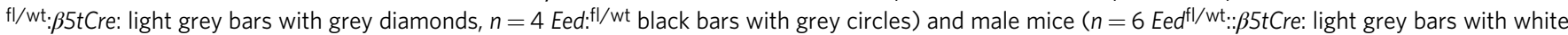
diamonds, $n=2$ Eed: ${ }^{f l / f l}$ black bars with white circles) at 4-6 weeks of age. Cellularities are displayed for each sex separately, TEC subset frequencies are

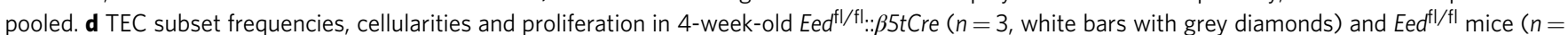
3 , grey bars with black circles). Representative histograms showing BrdU incorporation and frequencies of BrdU-positive cTEC and mTEC subsets. Data in graphs represent mean and standard deviations (SD) and are pooled from independent experiments $(\mathbf{a}, \mathbf{c})$ or display one experiment representative of two independent experiments $(\mathbf{b}, \mathbf{d})$. Contour plots $(\mathbf{a}, \mathbf{b})$ and histograms $(\mathbf{d})$ are representative of data in bar graphs. The gating for thymic epithelia is displayed in Supplementary Fig. 1. $n=$ biologically independent replicates per group. ${ }^{\star} p<0.05,{ }^{\star \star} p<0.01,{ }^{\star \star \star} p<0.001$ (two-tailed unpaired Student's $t$ test). Asterisks in red indicate comparisons between Eed $d^{f|f|::} \beta 5 t C r e$ and $E e d^{f l / f l}$ mice, whereas black and grey asterisks compare results from identical mouse strains at different time points. Source data including exact statistical test values are provided in the Source Data file.

and $\mathrm{CCR}^{-} \mathrm{TCR}^{+} \mathrm{DP}$ or CD4SP thymocytes) was undisturbed by the absence of EED in TEC (Fig. 4a). The second wave, which takes place in the medulla and is characterized by Helios expression on Foxp3 $3^{-}$CD4SP thymocytes ${ }^{27}$, was reduced (CD24 $4^{+}: 4.1 \pm 0.4 \%$ vs. $6.6 \pm 0.6 \%$; $\mathrm{CD}_{2} 4^{-}: 2.5 \pm 0.3 \%$ vs. $3.4 \pm 0.4 \%$, Fig. $\left.4 \mathrm{~b}\right)$. This reduction correlated with both lower CD5 expression on SP thymocytes (Fig. 4c) and significantly fewer apoptotic cells present in the Helios-positive subsets (Fig. 4d) suggestive of having received TCR-mediated signals of too low avidity to commit to programmed cell death ${ }^{28}$. In contrast, we observed no differences in thymocyte selection and maturation in Eed: $:^{\mathrm{fl} / \mathrm{wt}}: \beta 5 t$ Cre mice indicating that the presence of one Eed allele in TEC is sufficient to instruct regular T cell development (Fig. 4e).

Under physiological conditions, apoptotic cells are rapidly engulfed and cleared by thymic macrophages $(\mathrm{M} \varnothing)^{29}$ and conventional Type 1 DCs $(\mathrm{cDC} 1)^{30}$. Thymus immigrating DCs and $\mathrm{B}$ cells can also provide peripheral antigens for central tolerance induction ${ }^{9-11}$. We therefore determined the frequencies of thymic Mø, B cell and DC subsets in control and $E e d^{\mathrm{fl} / \mathrm{fl}}:$ : $\beta 5 t C r e$ mice and found that their relative frequency was significantly increased in mutant animals (Supplementary Fig. 4). Increased $\mathrm{Mø}$ and $\mathrm{cDC1}$ frequencies also indicated effective removal of apoptotic cells ${ }^{1,30,31}$ thus providing a cellular mechanism for the reduced detection of cells having received negative selection signals. In addition, the relative increase in B cells and thymus immigrating DCs could compensate for defects in selection mechanisms by PRC-deficient mTECs. Thus, the frequency of detectable negatively selected thymocytes was significantly reduced in the medulla, but not the cortex, of Eed $^{\mathrm{fl} / \mathrm{fl}}:: \beta 5 t$ Cre mice.

The TCR repertoire in single-positive thymocytes of $\mathrm{Eed}^{\mathrm{fl} / \mathrm{fl}}$ :: $\boldsymbol{\beta 5 t C r e}$ mice is narrowed. We extended the analyses of thymocyte selection by characterizing the TCR repertoire selected in the

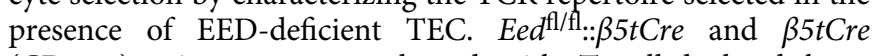
(CD45.2) mice were transplanted with $\mathrm{T}$ cell-depleted bone marrow cells from mice transgenic for the YAe62 TCR $\beta$ chain $(\text { TCRVb8.2) })^{32}$. Donor-derived (CD45.1) thymocytes at distinct developmental stages (see Supplementary Fig. 5a) were isolated 4 weeks after transplantation and their TCRa chain repertoire was sequenced. Each T cell lineage showed a high degree of TCR $\alpha$ repertoire overlap between mutant and control samples, and the $\mathrm{T}$ cell lineages were equally distinct from each other regardless of the PRC2-deficiency (Fig. 5a and Supplementary Fig. 5). Certain amino acid doublets and cysteine promote $\mathrm{T}$ cell self-reactivity when present at the apex of the complementarity-determining region 3 (CDR3) of the TCR $\alpha$ or TCR $\beta$ chain ${ }^{33-35}$. The frequency of TCR $\alpha$ clonotypes bearing these amino acid motifs varied between $\mathrm{T}$ cell lineages ${ }^{33-35}$ but did not differ significantly between mutant and control samples (Fig. 5b, c). Except for a distal shift in Traj segment usage by DP thymocytes in mutant mice, Trav and Traj gene segment usage and CDR3 length distributions were comparable to controls (Supplementary Fig. 5). This shift in Traj may reflect a longer sojourn in the cortex due to the presence of a larger cTEC scaffold relative to the thymocyte pool in EED-mutant mice. Whereas the diversity of the preselection and wave 1 TCRa repertoires was normal, the CD4SP and CD8SP TCRa repertoires selected by EED-deficient TEC showed a 2-fold lower diversity than controls (Fig. 5d, e). These results indicate that EED deficiency did significantly narrow the TCRa repertoires of CD4SP and CD8SP thymocytes.

Increased frequency of activated $T_{\text {reg }}$ cells in Eed $^{\mathrm{fl} / \mathrm{fl}}:: \beta 5 t C r e$ mice. The peripheral T cell compartment of $E e d^{\mathrm{fl} / \mathrm{fl}::: \beta 5 t C r e}$ mice demonstrated a $\mathrm{T}$ cell lymphopenia, equally affecting CD4 and CD8 T cells, (Fig. 6a, b). This resulted in a reduced frequency of naive and an expansion of central and effector memory $\mathrm{T}$ cells (Fig. 6c). Naive T cells from Eed ${ }^{\mathrm{f} / \mathrm{fl}}:: \beta 5 t$ Cre mice neither showed a deficiency in proliferation nor IL-2 production in response to $\mathrm{CD} 3 \mathrm{mAb}$ and co-stimulatory signals (Fig. 6d).

We next determined the frequency and activation state of $\mathrm{T}_{\text {reg }}$ cells. In comparison to controls, mutant mice showed a significant increase in the frequency of $\mathrm{T}_{\text {reg }}$ among CD4 cells (Fig. 6b) with a larger proportion of these cells displaying an activated phenotype, as revealed by a higher frequency of cells negative for CD62L (Fig. 6c) and positive for CD103 and/or ICOS (Fig. 6e). In comparison to control mice, peripheral $\mathrm{T}_{\text {reg }}$ cells from $E e d^{\mathrm{fl} / \mathrm{fl}}:: \beta 5 t C r e$ mice exerted a higher potency suppressing in vitro the proliferation of mitogenically activated CD4 effector cells (Fig. 6f). Taken together, these findings indicate that the increased frequency and higher suppressive capacity of $\mathrm{T}_{\text {reg }}$ cells in Eed $^{\mathrm{f} / \mathrm{fl}}:: \beta 5 t C r e$ mice likely control a dysregulated immune response as a result of either by lymphopenia or self-reactivity.

Eed $^{\mathrm{fl} / \mathrm{fl}}:: Z s G r e e n:: \beta 5 t C r e$ mice contain mTEC with PRC2 activity. TEC isolated from Eed: ${ }^{\mathrm{fl} / \mathrm{fl}}: \beta 5 t C r e$ mice contained, as noted above (Fig.1f), a subpopulation that had escaped gene deletion and thus maintained its H3K27me3 marks. These "EED escapees" were predominantly mTEC, a finding that was also

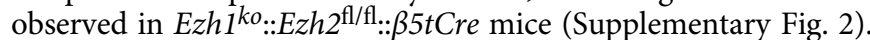
This result was unexpected as Cre expression under the Psmb11 transcriptional control targets $>99.5 \%$ of all mTECs $^{26}$. To exclude a lack of Cre activity as an explanation for the detection 
a

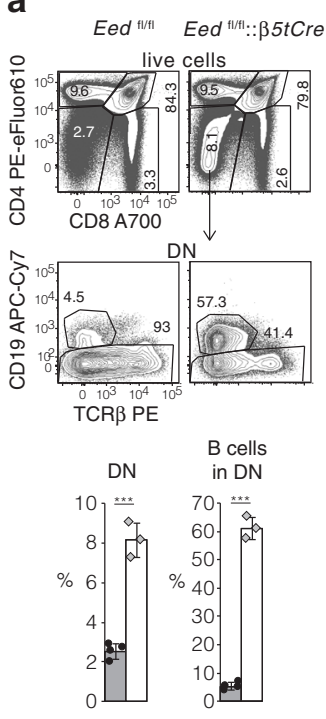

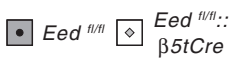

b
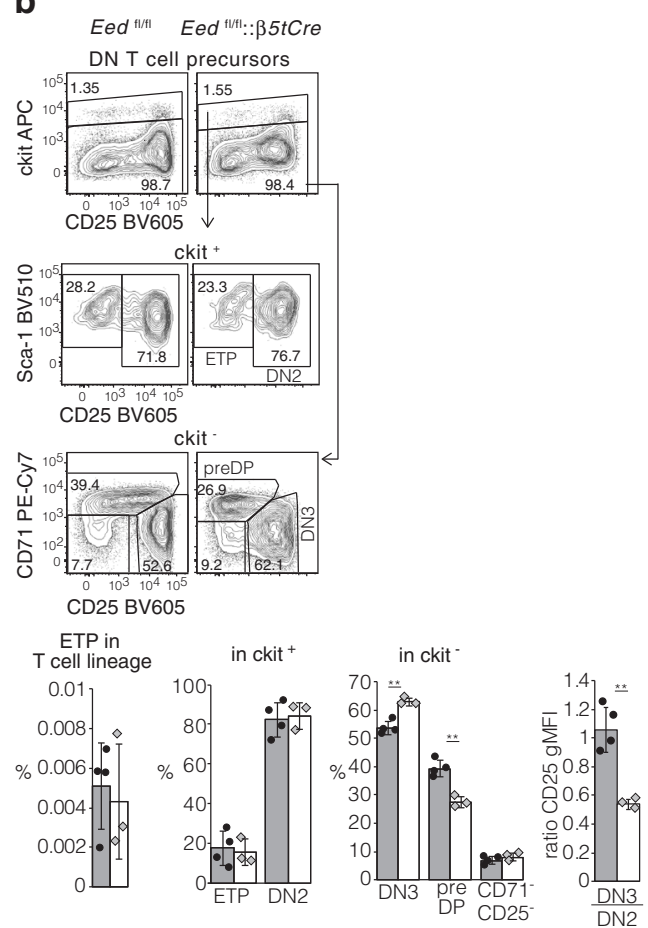

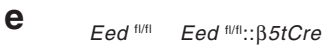

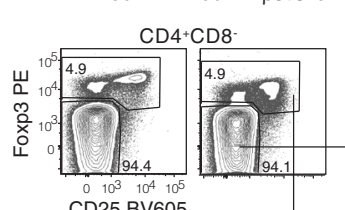
CD25 BV605
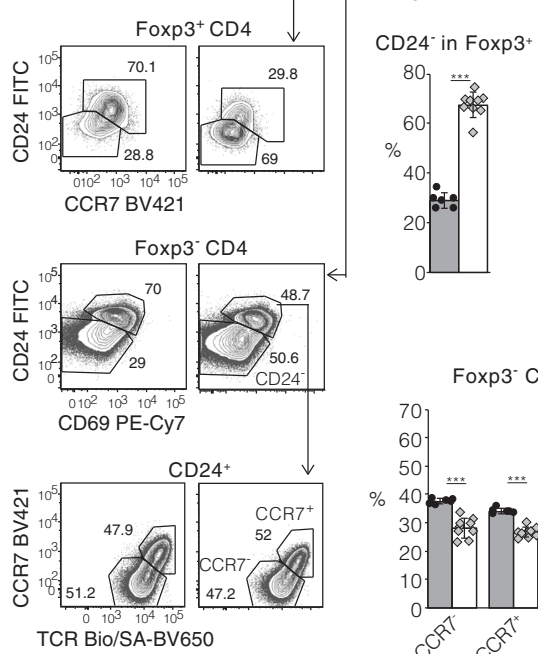

C
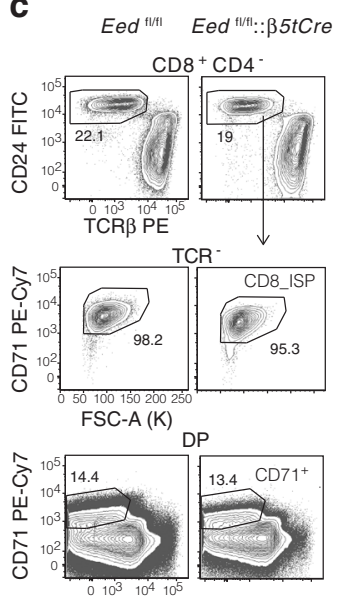

TCRB PE

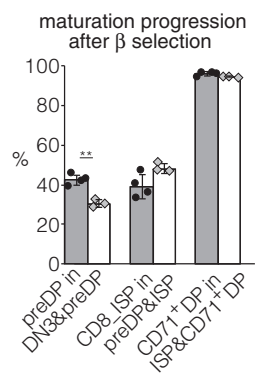

f re-circulated in CD24 SP
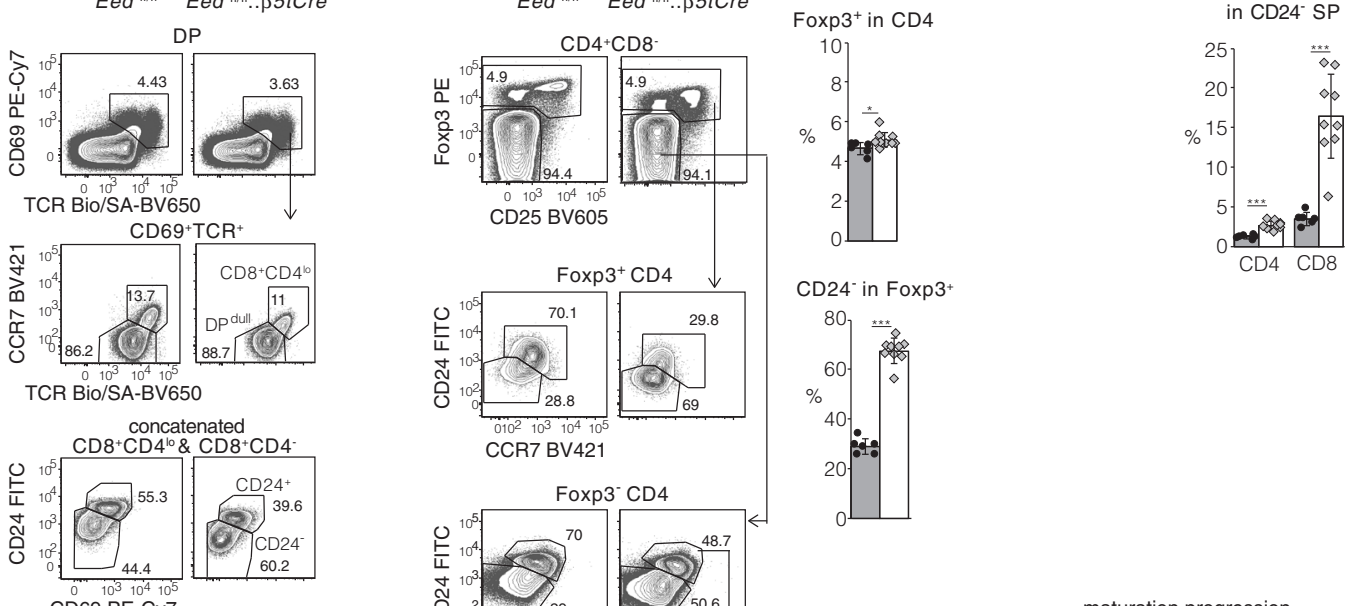

CD69 PE-Cy7
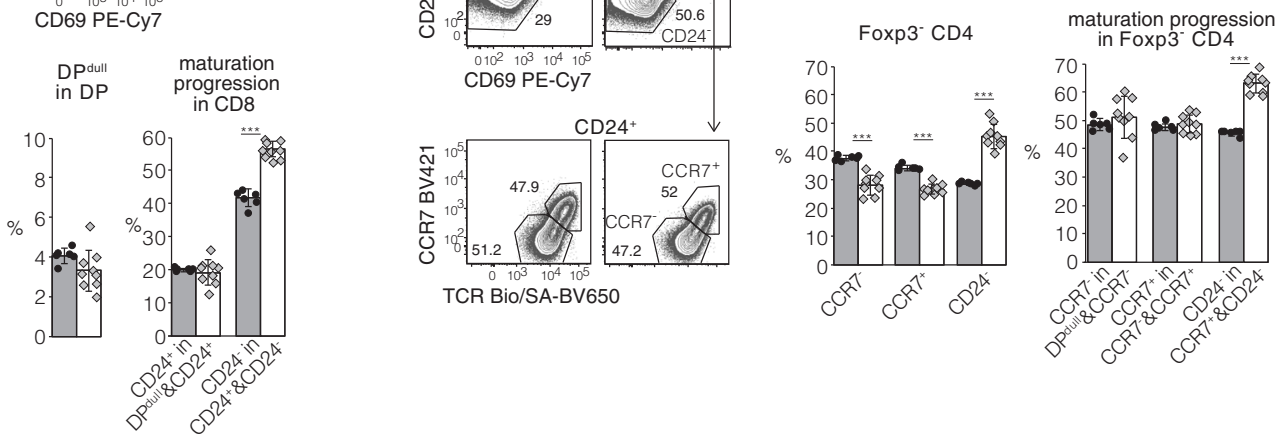

of mTEC with regular H3K27me3 marks, we next generated $E e d^{\mathrm{fl} /}$ fl::ZsGreen:: $\beta 5 t C r e$ mice where ZsGreen expression depends on the Cre-mediated removal of a floxed stop-cassette $5^{\prime}$ of the transgenic reporter. In these mice, $98.6 \pm 0.3 \%$ of all cTEC, yet only $80.3 \pm 5.6 \%$ of all mTEC expressed ZsGreen (Fig. 7a). The loss of ZsGreen expression coincided with the detection of $\mathrm{H} 3 \mathrm{~K} 27 \mathrm{me} 3$ marks in $93.2 \pm 2 \%$ of all TEC, whereas the vast majority of $\mathrm{ZsGreen}^{+}$TEC lacked H3K27me3 (97.4 $\pm 0.6 \%$; Fig. 7b) although their total histone $\mathrm{H} 3$ abundance was comparable (Fig. 7c). Furthermore, H3K27me2 median fluorescence 
Fig. 3 EED-deficient TEC affect thymocyte development at early and late stages. a Distribution of $B$ cells and T lineage precursors within DN cells. b Quantification of ETP, DN2, DN3 and preDP subsets within DN T cell precursors. c Analysis of maturational progression from CD8 ISP to CD71pos DP thymocyte stages. Characterization of the developmental progression within CD8 (d) and CD4 lineage (e) selected thymocytes. $\mathbf{f}$ Quantification of recirculated peripheral T cells within mature CD24neg SP subsets. Representative flow cytometry contour plots are shown, bar graphs display mean values

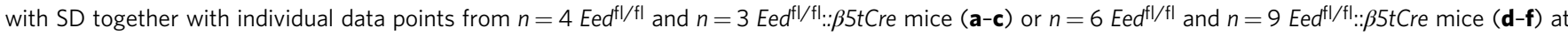
3 weeks of age. ${ }^{*} p<.05,{ }^{\star \star} p<.01,{ }^{\star \star *} p<.001$ (two-tailed unpaired Student's $t$ test). Data in bar graphs are from one experiment representative of three independent experiments. Eed ${ }^{f \mid / f l}: . \beta 5 t C r e:$ white bars with grey diamonds, Eed ${ }^{f l / f l}$ grey bars with black circles. $n=$ biologically independent replicates per group. ${ }^{\star} p<0.05,{ }^{\star \star} p<0.01,{ }^{\star \star \star} p<0.001$ (two-tailed unpaired Student's $t$ test). The gating for thymocyte subsets is displayed in Supplementary Fig. 3. Source data including exact statistical test values are provided in the Source Data file.

was drastically reduced in $\mathrm{ZsGreen}^{+}$but not $\mathrm{ZsGreen}^{-}$mTEC subsets (mTEClo: $\mathrm{ZsG}^{+} 8.5 \pm 3.0 \%$, $\mathrm{ZsG}^{-} 30.1 \pm 23.5 \%$, control $38.6 \pm 16.0 \%$; mTEChi: $\mathrm{ZsG}^{+} 13.7 \pm 5.2 \%, \mathrm{ZsG}^{-} 115.6 \pm 28.6 \%$, control $100 \pm 19.6 \%$; Fig. $7 d$, left panels). In addition, the lack of EED correlated with a 2 -fold reduction in EZH2 fluorescence (cTEC: $\mathrm{ZsG}^{+} 6.8 \pm 1.5 \%$, control $14.2 \pm 5.2 \%$; mTEClo: $\mathrm{ZsG}^{+}$ $10.2 \pm 2.9 \%$, control $18.6 \pm 2.0 \%$; mTEChi: $46.1 \pm 4.2 \%$, control $100 \pm 4.5 \%$; Fig. $7 \mathrm{~d}$, right panels). However, a minor fraction of $\mathrm{ZsGreen}^{+}$and $\mathrm{H} 3 \mathrm{~K} 27 \mathrm{me}^{+}$TEC were detected $(1.9 \pm 0.8 \%$; Fig. 7b) that were predominantly mTEC but displayed lower amounts of ZsGreen (Fig. 7b). This phenotype is compatible with promiscuous $\beta 5 t$ Cre expression in "EED mTEC escapees". Hence, most $\mathrm{H} 3 \mathrm{~K} 27 \mathrm{me} 3^{+}$"EED escapees" must be the progeny of rare TEC progenitors that do not express Cre.

Eed ${ }^{\mathrm{fl} / \mathrm{fl}}:: Z s G r e e n:: \beta 5 t C r e$ mice provided an opportunity to compare in the same thymus ZsGreen-positive EED-deficient and ZsGreen-negative EED-proficient TEC (aka "EED escapees"). ZsGreen-negative cTEC had a reduced Ly51 and MHC class II expression, thus providing a phenotype distinct from that of ZsGreen-positive cTEC (Fig. 7f). mTEC and the contingent of mature mTEChi demonstrated markedly reduced frequencies in the absence PRC2 activity in contrast to corresponding cells among "EED escapees" (Fig. 7e). The frequency of CD40 ${ }^{+}$EEDdeficient mTEChi remained unchanged but that of $\mathrm{AIRE}^{+}$cells was reduced when compared to controls (Fig. 7g). The maturational progression of EED-deficient mTEC thus progressively declined, whereas that of "EED escapees" was comparable to control mTEC. Both ZsGreen-positive and -negative mTEC were uniformly distributed throughout the medulla of $\mathrm{Eed}^{\mathrm{fl} / \mathrm{fl} \text { :: }}$ ZsGreen:: $\beta 5 t C r e$ mice, yet the latter cell type occasionally formed small cell clusters (Fig. $7 \mathrm{~h}$ ). Taken together, these results are compatible with the notion that EED-proficient mTEC had most likely arisen via a non-canonical differentiation path unrelated to ineffective Cre recombination.

EED-competent mTEC in $E e d^{\mathrm{fl} / \mathrm{fl}}:$ : $\beta 5 t C r e$ mice represent a novel, non-canonical mTEC differentiation pathway. In wildtype mice, mTECs are derived from progenitors that express Psmb1126. To test the hypothesis that "EED escapees" constitute a separate lineage, we next compared the transcriptome of ZsGreen-positive EED-deficient and ZsGreen-negative EEDproficient mTEChi. A principal component analysis of the gene expression profiles identified three distinct clusters with "EED escapees" mTEChi grouping separately from EED-deficient and

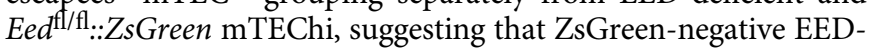
proficient cells either represent a distinct, non-canonical TEC lineage or a distinct differentiation pathway ending in a similar, but nonetheless transcriptomically distinct, medullary compartment (Fig. 8a). Both ZsGreen-positive and -negative mTECs showed marked transcriptomic differences to Eed ${ }^{\mathrm{fl} / \mathrm{fl}}:: Z s G r e e n$ mTECs (518 and 305 genes, respectively, significant at FDR < 0.05). There were also transcriptomic differences between ZsGreen-positive and -negative mTECs (664 genes, significant at FDR < 0.05; Supplementary Fig. 6 and Supplementary Data 1-3).
For example, genes encoding proteins involved in the immunoproteasome were expressed at lower copy numbers in the absence

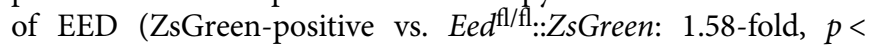
0.002; ZsGreen-positive vs. ZsGreen-negative: 2.66-fold, $p<$ 0.002 ; Fig. 8 b, c). Endopeptidase activity was particularly marked in the ZsGreen-negative mTECs, with the highest expression of 12 components of the proteasome seen in these cells. Conversely, ZsGreen-positive mTECs had elevated expression of genes within pathways regulating transcriptional activity related to cellular stress, such as Fosb and Jun (Fig. 8c). Moreover, genes that were differentially expressed between ZsGreen-positive and either ZsGreen-negative or Eed ${ }^{\mathrm{f} / \mathrm{fl}}$ mTECs were also enriched within molecular pathways associated with antigen processing and presentation (Supplementary Fig. 7). These results suggested a deficiency in normal antigen processing in the absence of EED expression along with upregulation of pathways indicative of cellular stress.

Differential gene expression in EED-deficient and EEDproficient mTEC of Eed $^{\text {fl/fll::ZsGreen:: } \beta 5 t C r e ~ m i c e . ~ m T E C ~ s u b-~}$ populations provide an almost complete molecular representation of TRAs with some of them being expressed at low frequency and in low copy number ${ }^{8}$. This capacity is aided by the transcriptional facilitator AIRE, which promiscuously regulates the expression of genes marked at their transcription start site (TSS) by $\mathrm{H} 3 \mathrm{~K} 27 \mathrm{me} 3^{8}$. We therefore determined at single-cell resolution whether EED-deficient and -proficient mTECs from $E e d^{\mathrm{fl} / \mathrm{f}}:$ : ZsGreen:: $\beta 5$ tCre mice maintained their normal transcriptional programmes. Single-cell analysis of cTECs showed a clear separation between EED-deficient and $E e d^{\mathrm{fl} / \mathrm{fl}}$ cells based on changes in transcripts associated with antigen processing and presentation (including Psmb11 and Ctsl; Supplementary Fig. 8). Significant differences were observed in the clustering centroids of each of the three separate mTEC $^{\text {hi }}$ populations analysed (all $p<0.05)$, further supporting the notion that EED-proficient mTEC in Eed ${ }^{\mathrm{fl} / \mathrm{fl}}:: Z s$ Green $:: \beta 5 t C r e$ mice represent the emergence of a non-canonical mTEC lineage (Supplementary Fig. 9).

Integration with existing single-cell mTEC transcriptomic datasets enabled us to further delineate mTEC differentiation in EED-deficient and -proficient mTEC $^{\text {hi }}$ (Fig. 9a, b) ${ }^{36,37}$. EEDdeficient mTEC $^{\text {hi }}$ from Eed ${ }^{\mathrm{fl} / \mathrm{fl}}:: Z s G r e e n:: \beta 5 t C r e$ mice were diverted away from mature mTEC into an immature, intertypical TEC phenotype (Fig. 9c, left and middle panel). Conversely, EEDproficient mTEC $^{\text {hi }}$ from Eed $d^{\mathrm{f} / \mathrm{fl}}:: Z s$ Green:: $\beta 5 t C r e$ mice were able to differentiate into mature mTEC similarly to $E e d^{\mathrm{fl} / \mathrm{fl}}$ mice but with a significant expansion of intertypical TEC (Fig. 9c, right panel). We used gene-regulatory network inference to identify transcription factor networks driving differences in mTEC differentiation and found Ascl1 and Irf7 network expression was significantly increased in EED-deficient mTEChi (Fig. 9d). Jun and Fos network expression was specifically elevated in EEDproficient mTEC $^{\text {hi }}$ from Eed ${ }^{\mathrm{fl} / \mathrm{fl}:: Z s G r e e n:: \beta 5 t C r e}$ mice relative to $E^{\mathrm{fl} / \mathrm{fl}}$ mice (Fig. 9e). This confirmed that EED-deficiency diverted mTEC differentiation towards a more immature 
a

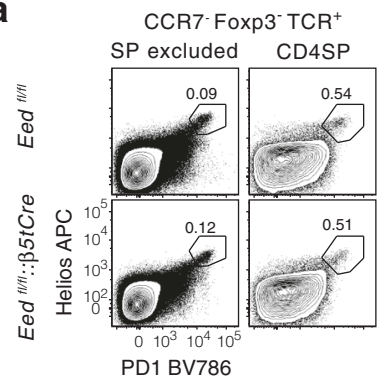

c

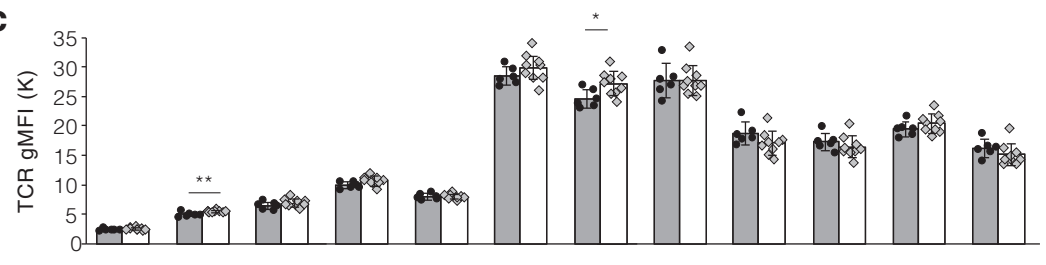

b

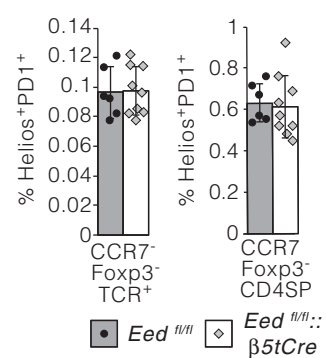

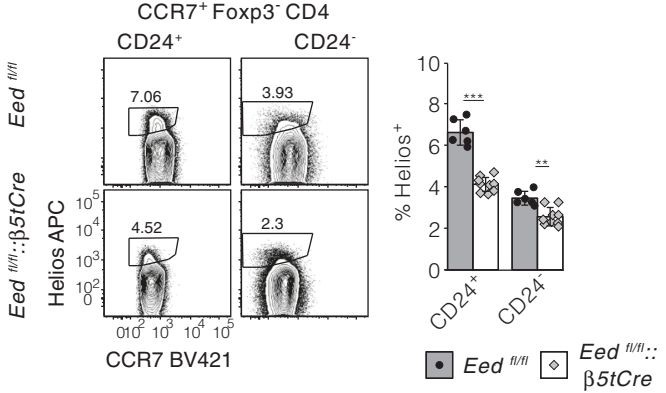

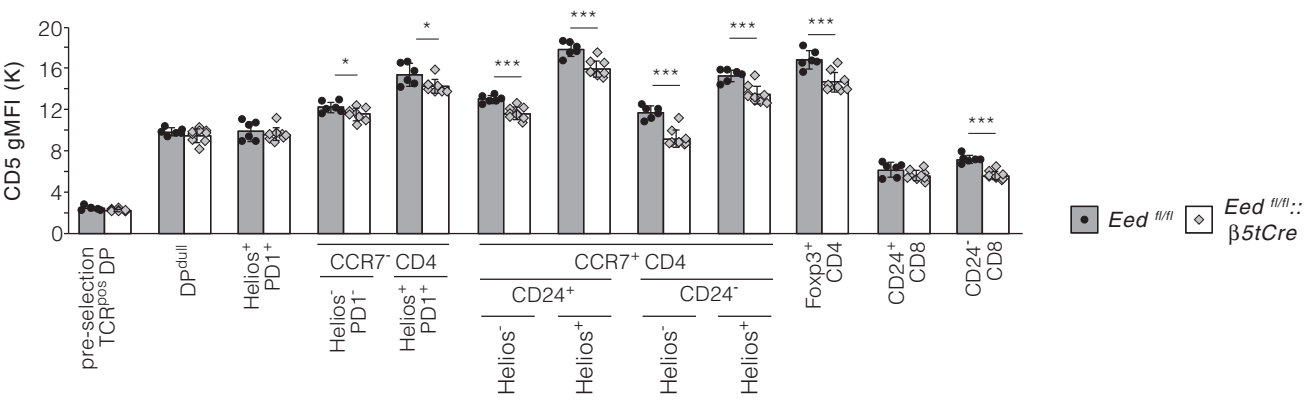

d
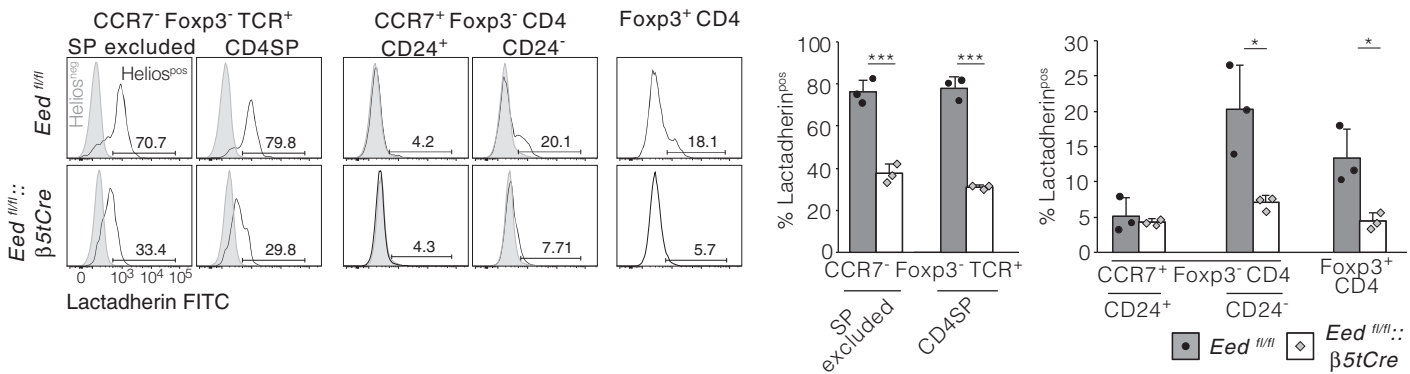

e
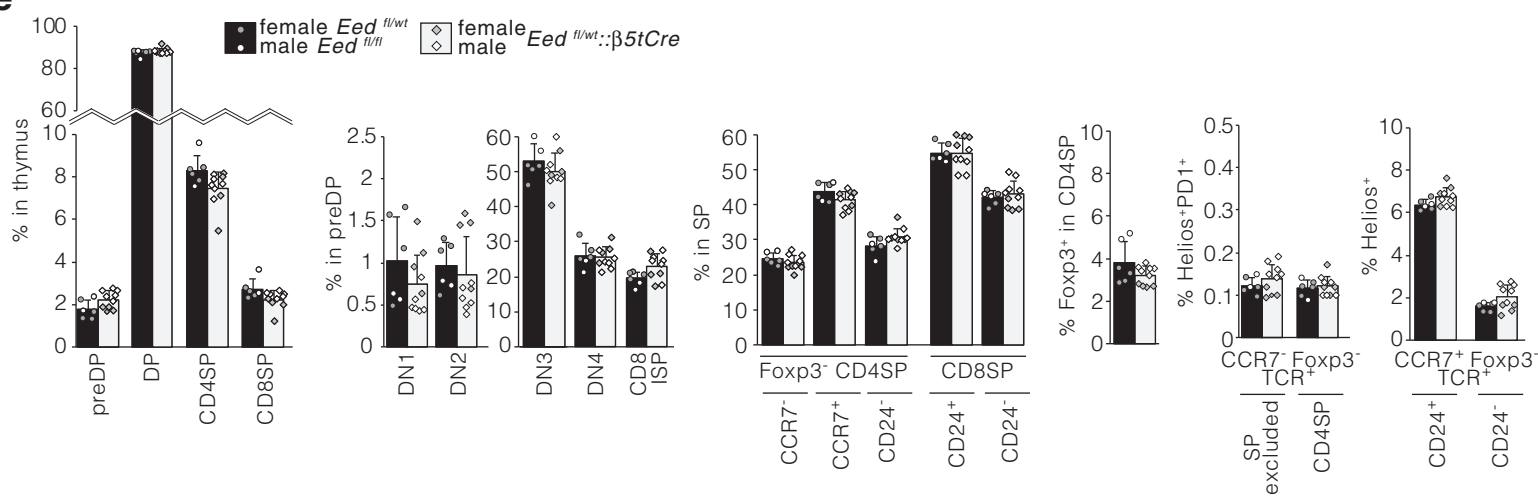

medullary phenotype, whereas the majority of "EED escapees", despite not originating from the canonical $\beta 5 t^{+}$TEC progenitor pool, were able to adopt a mature mTEC identity, although with several differences in transcription factor network expression.

We next assessed the consequences of the loss of EED on promiscuous expression of genes encoding TRAs whose transcripts depend on AIRE expression, are enhanced by AIRE or are generated independently of AIRE expression ${ }^{8}$. There were small changes in the overall expression of AIRE-controlled genes between EED-deficient and -proficient mTEC (Supplementary Fig. 10a). For example, AIRE-dependent TSPAN8 expression remained unchanged since this TRA was detected at comparable 
Fig. 4 Negative selection and TCR avidity are reduced in a EED-deficient thymic microenvironment. Analysis of negative selection of CD4 lineage thymocytes in the cortex (a) and the medulla (b). CD5 and TCR geometric mean fluorescence intensity on indicated thymocyte subsets (c). Quantification of apoptotic cells in Helios-positive thymocyte subsets (d). Analysis of thymocyte development in Eed:fl/wt: $\beta 5 t C r e$ and control mice. Frequencies of preDP, DP and SP subsets, developmental stages within preDP thymocytes, maturation within SP cells, frequencies of Treg in CD4SP and frequencies of negatively selected cells (e). Representative flow cytometry contour and histogram plots are shown. Bar graphs display mean values with SD together with individual

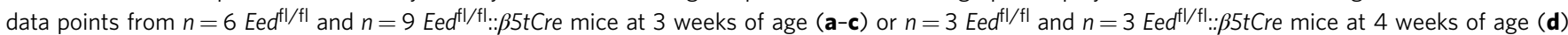
representative of at least two independent experiments. Eed $d^{f|f|}:: \beta 5 t C r e:$ white bars with grey diamonds, Eed ${ }^{f l / f l}$ grey bars with black circles. Data in $(\mathbf{e})$ are

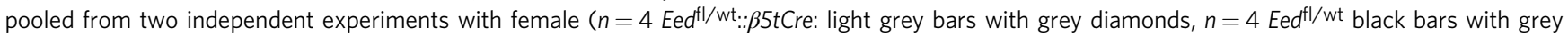

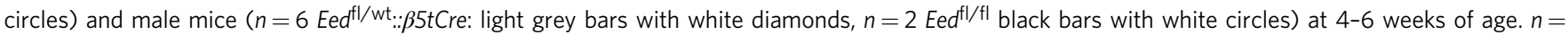
biologically independent replicates per group. ${ }^{\star} p<0.05,{ }^{\star \star} p<0.01,{ }^{\star \star \star} p<0.001$ (two-tailed unpaired Student's $t$ test). The gating for thymocyte subsets is displayed in Supplementary Fig. 3. Source data including exact statistical test values are provided in the Source Data file.

a

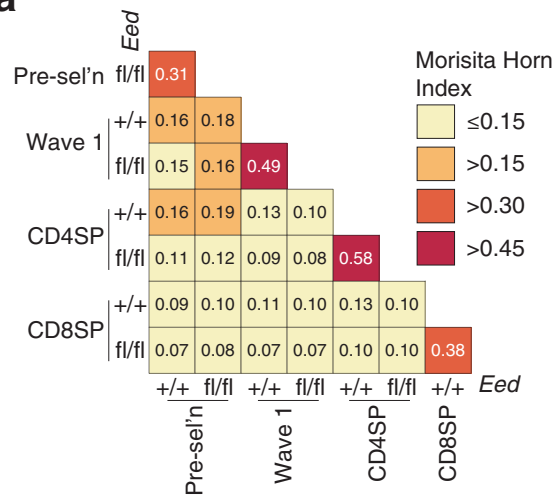

b

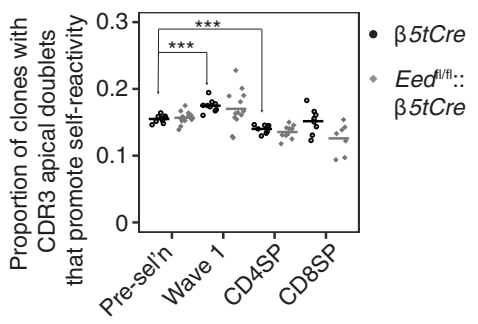

d

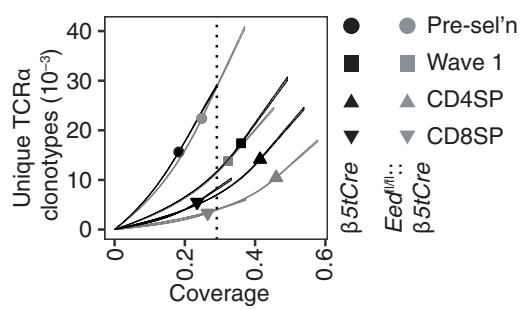

C

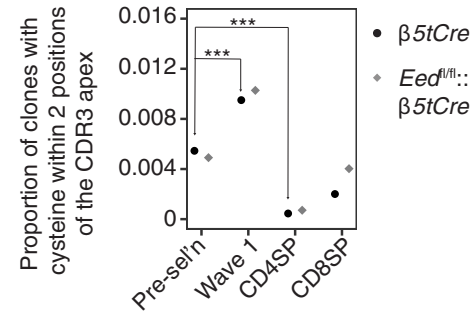

e

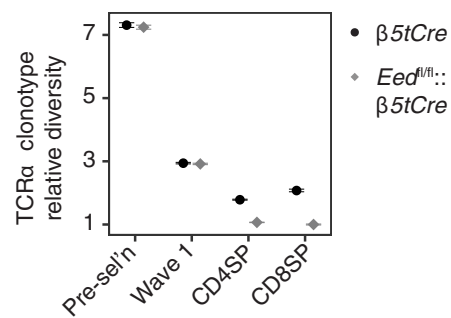

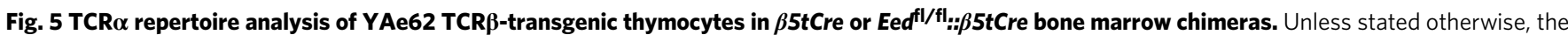
analyses compared TCR catalogues, which were formed by aggregating samples of a given T cell subset/genotype combination. The $\beta 5 t C r e$ and Eedfl/fl:: $\beta 5$ tCre TCR catalogues contained the following numbers of samples: 9 and 14 mice, respectively (pre-selection, Wave 1 and CD8SP); 8 and 14 mice, respectively (CD4SP). a Heatmap shows the Morisita-Horn index for each pair of TCR catalogues. b, c Amino acid composition of the CDR3 apex. Considering the amino acid doublets at CDR3 positions 6 and 7, (b) shows the proportion of TCR $\alpha$ clones with doublets that promote self-reactivity ${ }^{33}$. Each symbol represents an individual sample. Samples with fewer than 100 clones were excluded, (c) depicts the proportion of TCR $\alpha$ clones with cysteine anywhere from CDR3 position -2 to +2 as determined by apex-out CDR3 alignment ${ }^{35}{ }^{\star \star \star}{ }^{\star \star} p=4.3 \times 10^{-5}$ (pre-selection vs. Wave 1 ) and $p=1.7 \times 10^{-6}$ (pre-selection vs. CD4SP) using Student's $t$ test (unpaired, two-sided, Bonferroni-corrected) in (b) or $p=1.5 \times 10^{-6}$ (pre-selection vs. Wave 1 ) and $p=$ $2.8 \times 10^{-15}$ using Fisher's exact test ( $2 \times 2$ table, two-sided, Bonferroni-corrected $)$ in (c). d, e TCR $\alpha$ clonotype diversities. $\mathbf{d}$ Cumulative number of unique TCR $\alpha$ clonotypes plotted as a function of "coverage", defined as the proportion of all clones (encountered and unencountered) in a given T cell subset that belong to clonotypes encountered in the catalogue. Symbols show the observed values and curves outline the $95 \%$ confidence intervals of the rarefaction and extrapolation curves determined using 10 bootstrap replications. e Relative diversity of TCR catalogues. Symbols indicate ratios of the numbers of unique TCR $\alpha$ clonotypes per TCR catalogue at the level of coverage indicated by the vertical dashed line in (d). Error bars in (e) show the $95 \%$ confidence intervals of the relative diversities of TCR catalogues based on 10 bootstrap replications.

level in ZsGreen-positive and -negative mTEChi of experimental mice and their controls (Fig. 10a). By randomly sampling AIRE expressing single cells, we estimated for each of the different mTEC populations the number of detectable genes encoding TRAs (Fig. 10b). The breadth of promiscuous TRA expression was reduced in EED-deficient mTECs, affecting particularly AIRE-controlled loci (AIRE-dependent: 0.86 -fold, $p<0.0001$; AIRE-enhanced: 0.96-fold, $p<0.0001$; AIRE-independent TRA: 0.98 -fold, $p<0.0001$; all other genes: 0.99 -fold, $p<0.0001$ ). A similar change in the pattern of TRA expression was observed in ZsGreen-negative mTEC isolated from Eed $d^{\mathrm{f} / \mathrm{fl}}:: Z s G r e e n:: \beta 5 t C r e$ mice (AIRE-dependent: 0.84-fold, $p<0.0001$; AIRE-enhanced: 0.94-fold, $p<0.0001$; AIRE-independent TRA: 0.97 -fold, $p<$ 0.0001 ; all other genes: 0.99 -fold, $p<0.0001)$. Hence, there was a subtle reduction in the breadth of promiscuous gene expression in mTEChi proficient or deficient in PRC2 activity within the same thymic environment. However, multivariate analysis showed that the presence of H3K27me3 marks near the TSS predicted whether or not a gene was upregulated in EED-deficient mTECs relative to those that were EED-proficient independently of whether or not that gene was regulated by AIRE (Supplementary Fig. 10b). This finding suggests that at least a portion of the mild deficiency in promiscuous gene expression was more likely to be driven by thymic microenvironmental changes than a direct effect of EED-

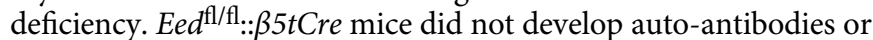
overt organ infiltration nor did they reveal signs of autoimmunity after antibody-mediated $\mathrm{T}$ cell depletion and subsequent endogenous $\mathrm{T}$ cell reconstitution (Supplementary Fig. 11). 
a

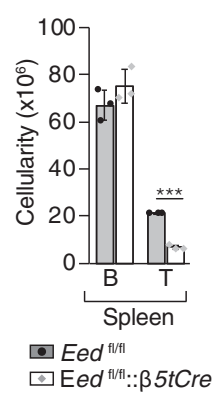

b

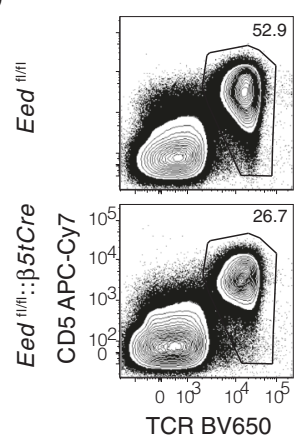

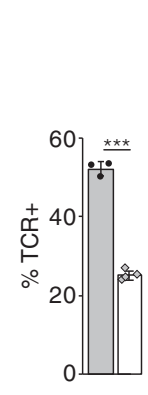

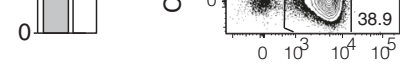
CD8 A700

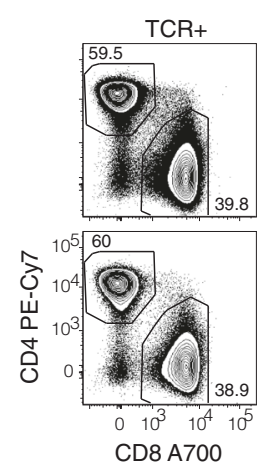

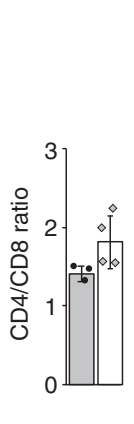

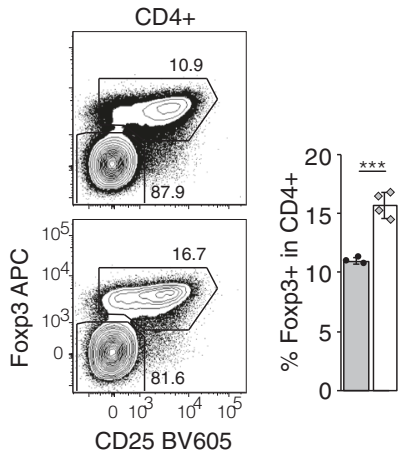

- Eed ${ }^{\text {flifl }}$ Eed $^{\mathrm{fl} / \mathrm{fl}:}:: \beta 5 t$ Cre
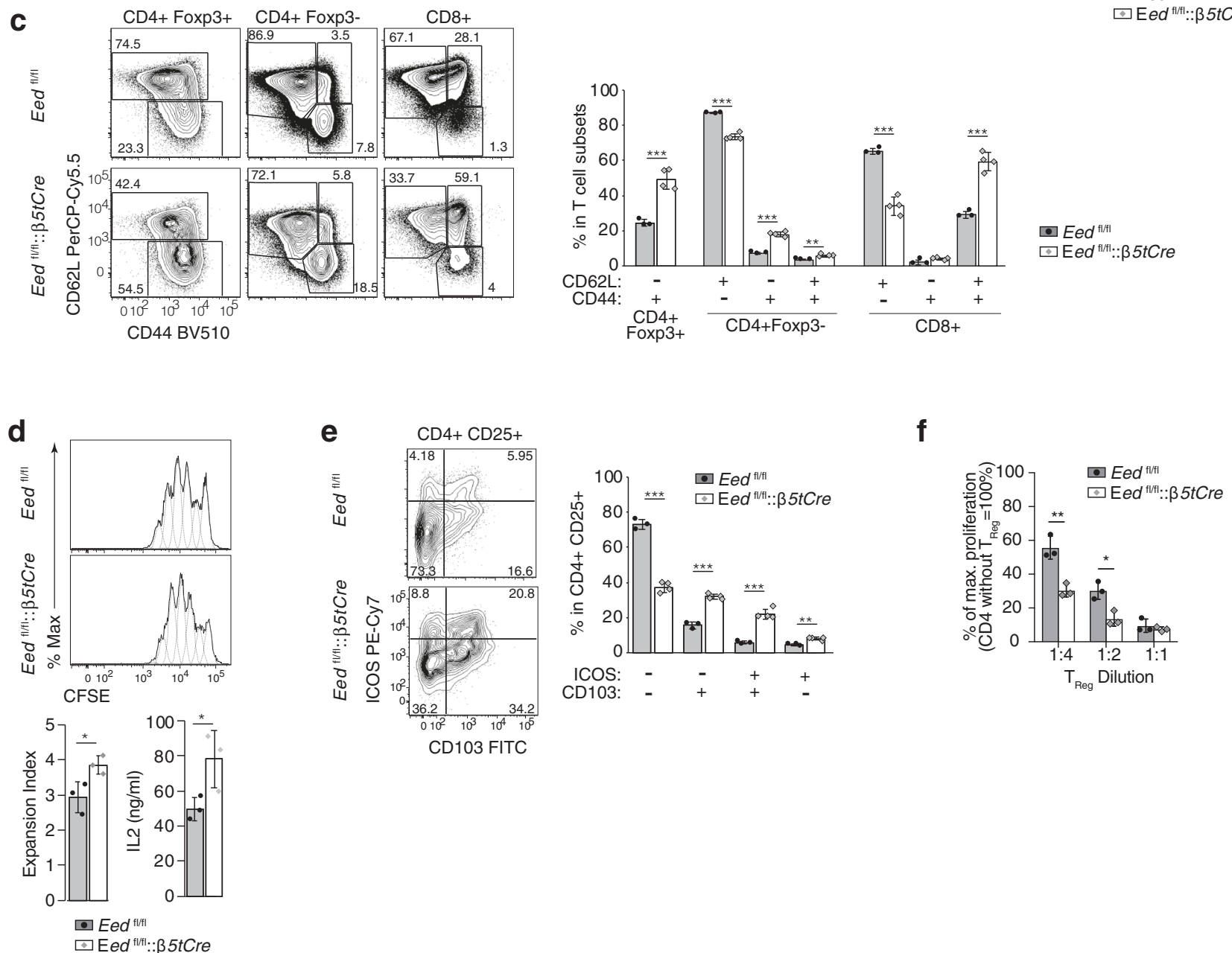

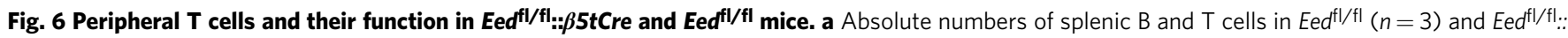
$\beta 5 t$ Cre mice $(n=3)$ at 4 weeks of age. $\mathbf{b}$ Representative contour plots and frequencies of lymph-node-derived T cell subsets. Frequency of TCR $\beta+T$ cells, the CD4/CD8 ratio within TCR $\beta+T$ cells and percentage of Foxp3 ${ }^{+} \mathrm{T}_{\text {Reg }}$ in CD4 T cells. c Frequencies of activated cells (CD44 $\left.{ }^{+} \mathrm{CD}_{2} 2 \mathrm{~L}^{-}\right)$in Foxp3 ${ }^{+} \mathrm{T}_{\text {Reg }}$ and frequencies of naive $\left(\mathrm{CD} 44^{-} \mathrm{CD}_{2} \mathrm{~L}^{+}\right)$, central memory $\left(\mathrm{CD} 44^{+} \mathrm{CD} 62 \mathrm{~L}^{+}\right)$and effector memory $\left(\mathrm{CD} 44^{+} \mathrm{CD} 62 \mathrm{~L}^{-}\right) \mathrm{T}$ cells within the populations of

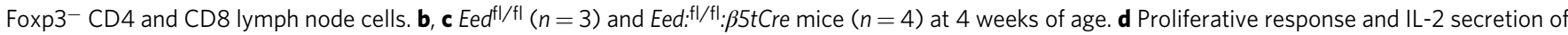
naive lymph node CD4 T cells $\left(C D 44^{l}{ }^{\circ} \mathrm{CD} 25^{-}\right)$from $\operatorname{Eed}^{\mathrm{fl} / \mathrm{fl}}(n=3)$ and Eed ${ }^{\mathrm{fl} / \mathrm{fl}::} \beta 5 \mathrm{tCre}$ mice $(n=3)$ at 4 weeks of age. Representative histograms of CFSE dilution (including model components used for expansion index calculations; grey dotted lines) following stimulation for three days with CD3 mAb (1 $\mu \mathrm{g} /$ $\mathrm{ml}$ ) and irradiated Rag2-/- splenocytes. Bar graphs display the mean expansion index (left) and mean IL2 concentrations in the supernatant (right) with

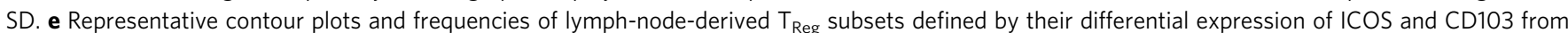
Eed $^{f|/ f|}(n=3)$ and Eed ${ }^{f l / f l:: \beta 5 t C r e ~ m i c e ~}(n=4)$ at 4 weeks of age. $\mathbf{f}$ T cell suppression assay: CD3 mAb-induced proliferation of sorted naive WT CD4

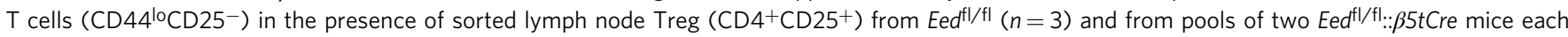
$(n=3)$ of mixed sex at 7-9 weeks of age. ${ }^{\star} p<0.05,{ }^{\star \star} p<0.01,{ }^{\star \star \star} p<0.001$ (unpaired Student's $t$ test). Data in graphs show mean \pm SD and are representative of two independent experiments. Contour plots $(\mathbf{b}, \mathbf{c}, \mathbf{e})$ and histograms $(\mathbf{d})$ are representative of data in bar graphs. Eed ${ }^{f / f f l::} \beta 5 t C r e:$ white bars with grey diamonds, Eed ${ }^{f l / f l}$ grey bars with black circles. $n=$ biologically independent replicates per group. Source data including exact statistical test values are provided in the Source Data file. 
a
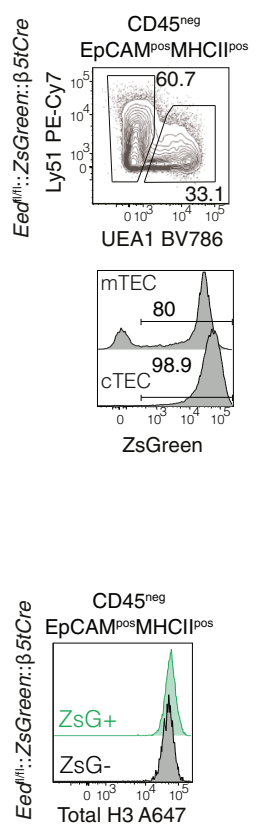

b

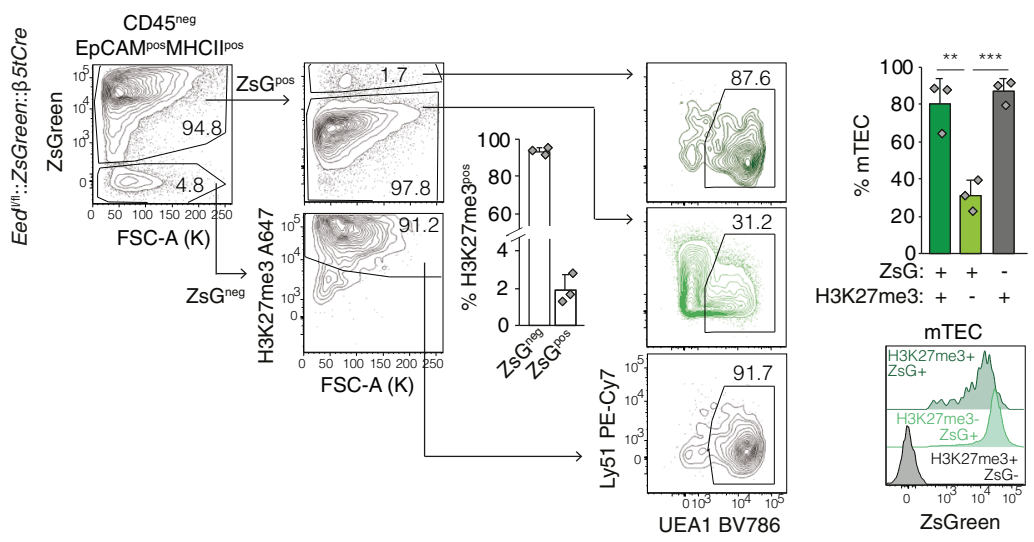

d
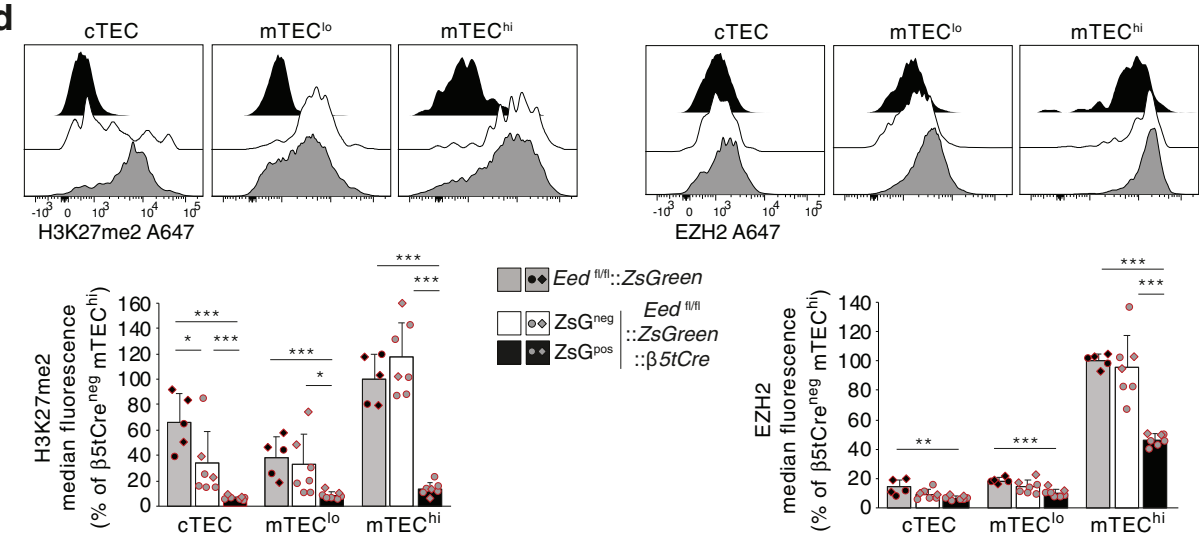

e

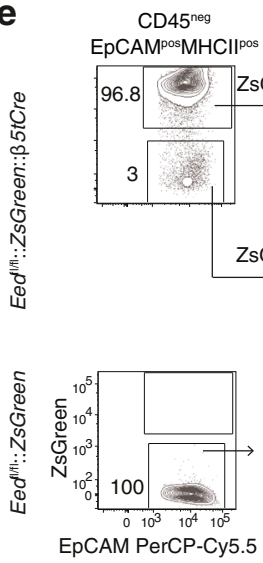
EpCAM ${ }^{\text {pos }} \mathrm{MHCI}^{\mathrm{pos}}$

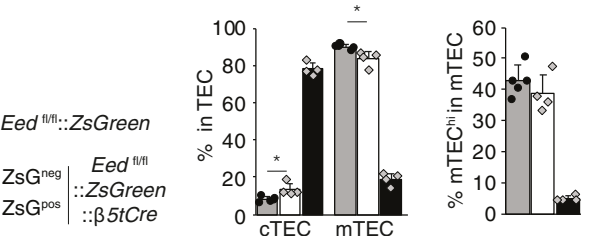

f
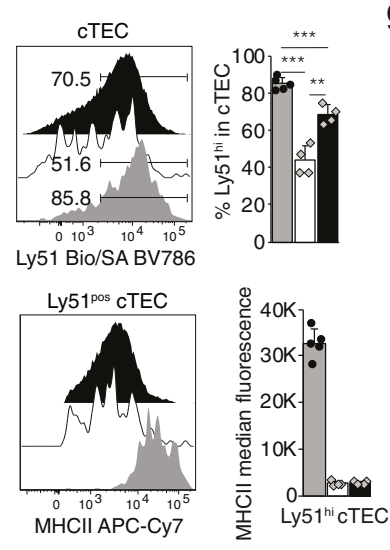

g
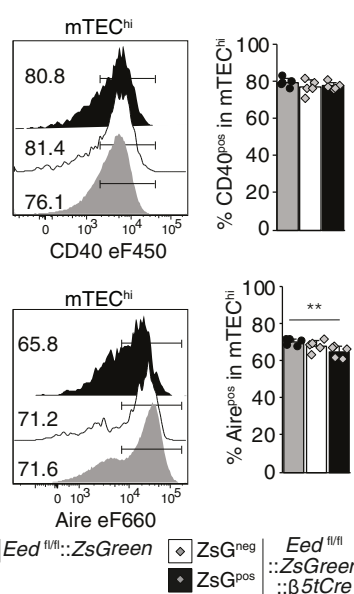

h

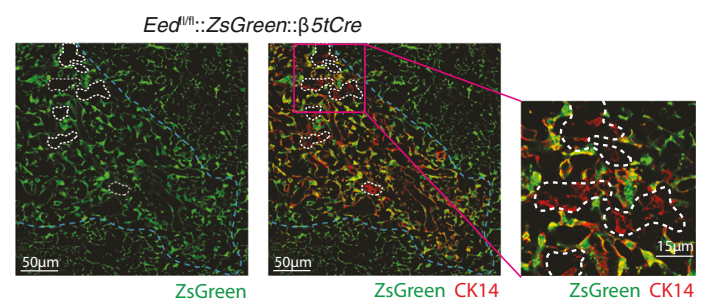

expressed in ZsGreen-positive mTECs than in either of the two other TEC populations (H3K27me3 ChIP/input signal within 4 $\mathrm{kb}$ of the TSS: ZsGreen-positive vs. ZsGreen-negative: median 2.36-fold, FDR $<0.0001 ; Z$ ZsGreen-positive vs. Eed ${ }^{\text {ft/fl }}$ median 2.14-fold, FDR $<0.0001$; Fig. 10c). Differences in the corresponding H3K27me3 ChIP-seq signals were, however, not observed 
Fig. 7 Eed ${ }^{f l / f l}:: Z s G r e e n: ; 5$ tCre mice contain a subpopulation of Eed-proficient $\mathbf{m T E C}$. a ZsGreen expression in cTEC and mTEC isolated from 4-week-old

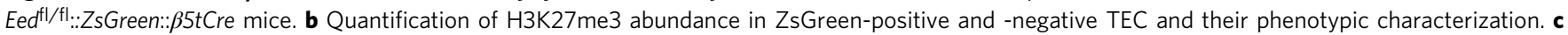
Representative histograms of histone $\mathrm{H3}$ abundance in ZsGreen-positive and -negative TEC. d Representative histograms and median fluorescence quantification of $\mathrm{H} 3 \mathrm{~K} 27 \mathrm{me} 2$ and EZH2. Fluorescence of control mTEChi was set as $100 \%$. Data are pooled from two independent experiments identified by circles and diamonds, respectively. e Representative FACS plots and quantitative analysis of cTEC, mTEC and mTEChi frequencies within ZsGreen-positive and -negative TEC. $\mathbf{f}, \mathbf{g}$ Representative histograms and quantitative analysis of ZsGreen-positive and -negative cTEC (f) and mTEChi ( $(\mathbf{g})$ phenotypes. $\mathbf{h}$ Spatial distribution of ZsGreen-positive and -negative TEC in thymus tissue sections of 4-week-old Eed ${ }^{f / f \mid}:: Z s G r e e n:: \beta 5 t C r e$ mice. Immunofluorescence analysis of mTEC expressing ZsGreen and cytokeratin 14 (CK14) in the medulla (marked by dashed blue line) identifying single cells and small aggregates of ZsGreen-negative cells (white dashed lines). Data in bar graphs show mean \pm SD and are pooled from two independent experiments (a, $\mathbf{b}$, $\mathbf{d}$ ) or are representative of two independent experiments $(\mathbf{e}-\mathbf{g})$. Contour plots and histograms are representative for data displayed as frequencies in bar graphs (a, $\mathbf{b}, \mathbf{d}, \mathbf{e}-\mathbf{g})$. Immunofluorescence data are representative of two independent experiments with 3 sections each. $\mathbf{a}, \mathbf{b}$ Eed ${ }^{f \mid / f l}:: Z s G r e e n:: \beta 5 t C r e(n=3)$, in $(\mathbf{b})$ dark green represents ZsGreen-positive H3K27me3-positive, light green shows ZsGreen-positive H3K27me3-negative, and grey depicts ZsGreen-negative

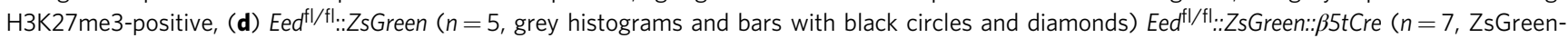
negative: white histograms and bars with grey circles and diamonds, ZsGreen-positive: black histograms and bars with grey circles and diamonds), (e-g)

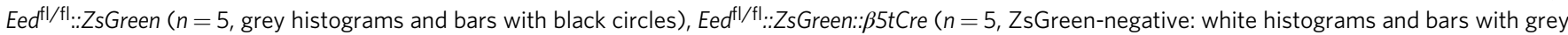
diamonds, ZsGreen-positive: black histograms and bars with grey diamonds); $n=$ biologically independent replicates per group, mice were 3-4 weeks of age. ${ }^{\star} p<0.05,{ }^{\star \star} p<0.01,{ }^{* \star} p<0.001$ (two-tailed unpaired Student's $t$ test). Source data including exact statistical test values are provided in the Source Data file.

between ZsGreen-negative and Eed fl/fl $^{\text {mTEC }}(\mathrm{FDR}>0.05)$. Taken together, these data demonstrated that the loss of EED early in TEC development resulted in an mTEC compartment with a relatively modestly reduced capacity for promiscuous gene expression. This finding suggests that the expression of TRAs is not critically dependent on repressive chromatin modifications.

\section{Discussion}

TEC development and function are dependent on EED, an essential component for assembly and activity of the PRC2 complex. The loss of EED in $\beta 5 t$-expressing TEC precursors and their progeny resulted in an absence of PRC2 activity in practically all cTEC (with the notable exception of few non-recombined cTEC displaying an atypical phenotype) and the majority of mTEC. However, up to $15 \%$ of all mTEC present in Eed ${ }^{\mathrm{fl} / \mathrm{fl}}:$ : $\beta 5 t C r e$ mice arise from an alternative mTEC developmental pathway originating from cells other than bi-potent, $\beta 5$ t-positive precursors and characterized by a distinct transcriptome, representing either a distinct TEC lineage or an alternate differentiation pathway into canonical mTEC cell types within an abnormal thymic microenvironment ${ }^{26,38}$. EED-proficient mTEC exhibits in Eed $^{\mathrm{fl} / \mathrm{fl}:: \beta 5 t C r e}$ mice a competitive growth/differentiation advantage over EED-deficient mTEC.

Despite the severely reduced cellularity of ETP and a partial block in $\beta$-selection, cTECs of Eed ${ }^{\mathrm{f} / \mathrm{fl}}:: \beta 5 t C r e$ mice support the differentiation to DP thymocytes and their positive selection despite differences in the expression of many genes required for antigen processing and presentation. Any deficiencies in antigen presentation may likely be compensated by the decreased thymocytes-to-cTEC ratio, thus lessening the competition between thymocytes and the normally limiting numbers of cTEC. This will result in an increased cross-talk between these two cell types and a longer sojourn of thymocytes in the cortex ${ }^{39}$. Indeed, the subtle differences in the choice of Traj segments within the TCRa repertoire of $E e d^{\mathrm{fl} / \mathrm{fl}:: \beta 5 t C r e}$ mice support this explanation as reflected by a more frequent use of distally positioned Traj family members ${ }^{40}$. Furthermore, the transition of thymocytes across the corticomedullary zone may be secondary to the decrease in Psmb11 expression, as observed in $\beta 5$ t-deficient mice ${ }^{41}$.

The exact timing of the loss of EED expression in TEC determines thymus organogenesis. In a recently described mouse

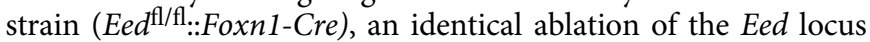
targeted to a time point early during TEC fate specification but prior to Psmb11 expression hinders TEC maturation resulting in a severely dysplastic thymus in which PRC2's role in development and function cannot be interrogated in detail ${ }^{42}$. Thus, it remains unknown whether mTEC development bypassing $\beta 5 \mathrm{t}$-positive bi-

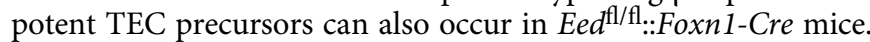
We therefore conclude that the loss of EED expression early during TEC differentiation (i.e. before the emergence of bi-potent $\beta 5 t$-positive TEC precursors) and the consequent loss of canonical gene silencing by PRC2 precludes not only the normal progression along the canonical TEC differentiation pathway but also excludes the emergence of the alternative mTEC pathway described here.

In addition to intrathymic B cells, Eed $d^{\mathrm{fl} / \mathrm{fl}:: \beta 5 t C r e}$ mice also show increased frequencies of thymic macrophages and DCs, although it is possible that this could simply be due to the observed reduction of thymocytes. The impaired antigen presentation by TEC may be compensated by immigrating DCs and $\mathrm{B}$ cells thus contributing to establishing central $\mathrm{T}$ cell tolerance ${ }^{9-11}$. Antigen transfer from mTEC to DCs may furthermore offset defects in mTEC-mediated antigen presentation ${ }^{43}$ and selecting a TCR repertoire with sufficient self-tolerance.

Signs of autoimmunity are not detected in eed $^{\mathrm{fl} / \mathrm{fl}}:: \beta 5 t$ Cre mice despite an impaired expression of antigen processing pathways, a limited reduction in the representation of TRA in MTEC, a narrowed breadth of the TCR repertoire and peripheral T cell lymophopenia. Although reliant on mTEC and DCs for their selection ${ }^{44-46}, \mathrm{~T}_{\text {reg }}$ generation is not offset by the reduced mTEC cellularity, indicating a likely compensation by the increased frequency of intrathymic DCs. The heightened frequency and function of peripheral $\mathrm{T}_{\text {reg }}$-resulting from lymphopenia-induced proliferation-contribute further to the observed lack of autoimmunity. Because peripheral $\mathrm{T}_{\text {reg }}$ cannot compensate for AIRE deficiency, the absence of autoimmunity corroborated our finding that EED deficiency results only in a minor alteration of TRA representation ${ }^{47}$.

Gene expression profiling of EED-deficient mTEChi demonstrated a significant upregulation of genes that are normally associated with $\mathrm{H} 3 \mathrm{~K} 27 \mathrm{me}$ marks in EED-proficient mTEChi, both wild-type and "EED escapees", a finding consistent with the removal of canonical gene silencing by PRC2. Changes in TEC development and composition are thus explained by gene derepression in pathways involved in epithelial differentiation as highlighted in other organs, such as lung, including Foxp1 and Nfib (Supplementary Fig. 7). This mechanism of de-repression was previously suspected as known PRC2 targets are characterized by active enhancer marks in immature but not mature mTEC $^{48}$. The current study demonstrates that these chromatin changes are not 
a

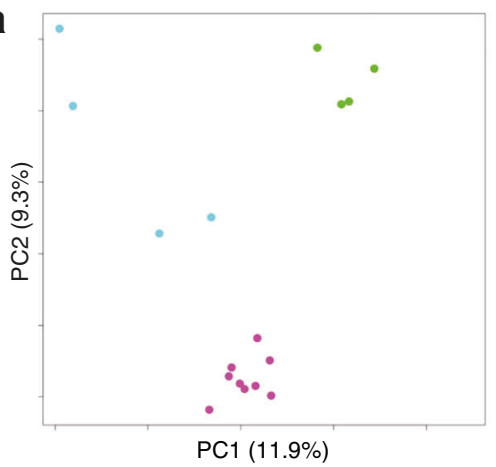

$\square$ Eed $^{\mathrm{fl|f|}}$

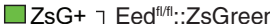

$\square$ ZsG- $\quad:: \beta 5$ tCre
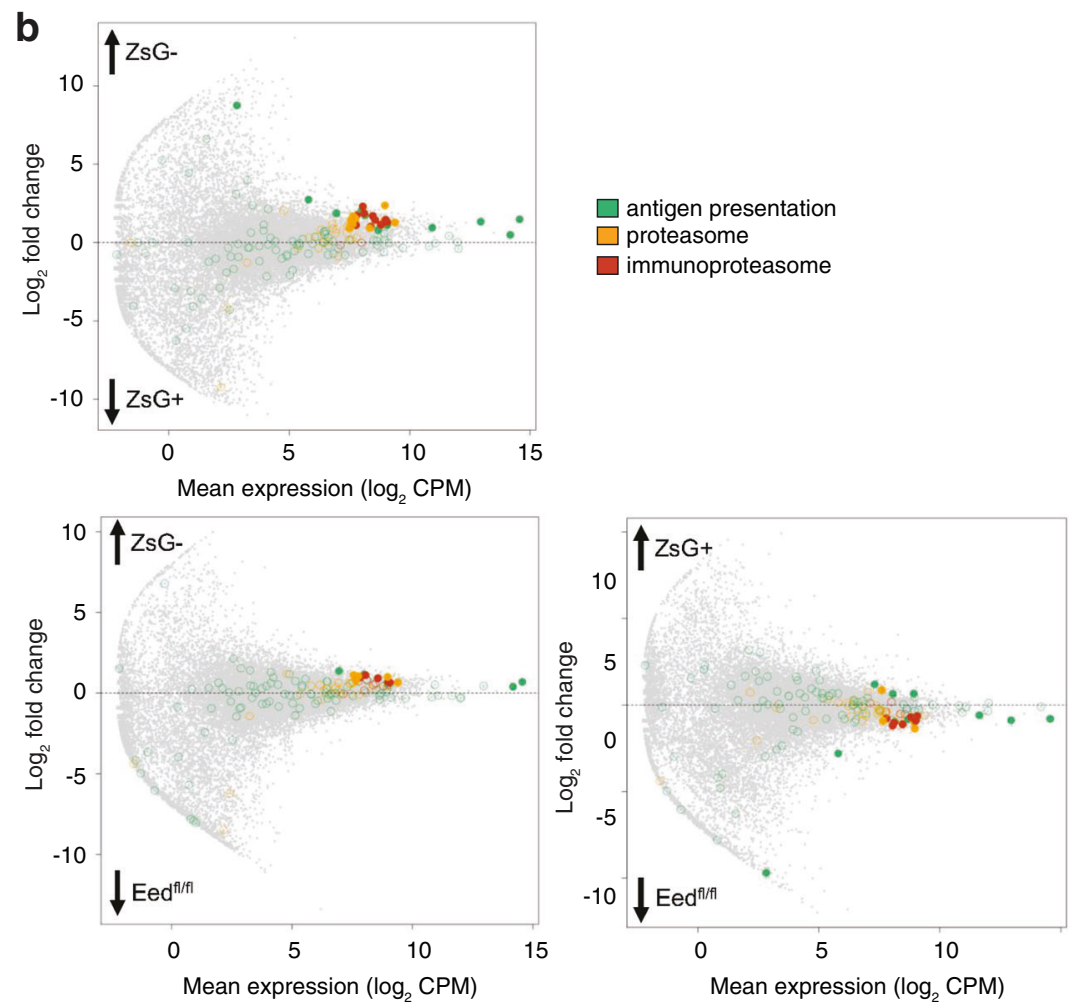

antigen presentation

proteasome

immunoproteasome
Mean expression $\left(\log _{2} \mathrm{CPM}\right)$

C

Structural constituent of ribosome GO:0003735 rRNA binding GO:0019843

Threonine - type endopeptidase activity GO:0004298

Threonine - type peptidase activity GO:0070003 NADH dehydrogenase activity GO:0003954

Enzyme inhibitor activity GO:0004857

Glycosaminoglycan binding GO:0005539

Heparin binding GO:0008201

RNA polymerase II cis - regulatory region... GO:0000978

DNA - binding transcription activator activity, RNA... GO:0001228

DNA- binding transcription activator activity GO:0001216

Peptidoglycan binding GO:0042834

Oligosaccharide binding GO:0070492

Receptor ligand activity GO:0048018

Signalling receptor activator activity GO:0030546

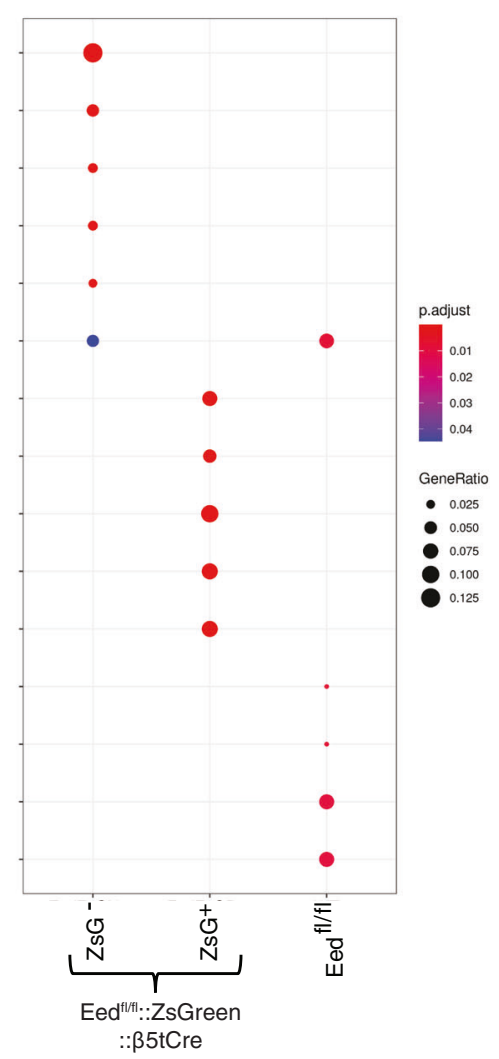


a

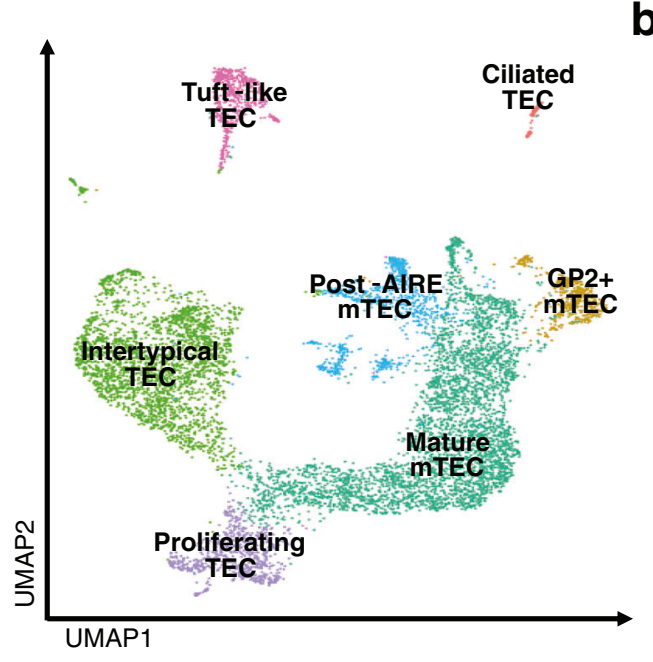

b

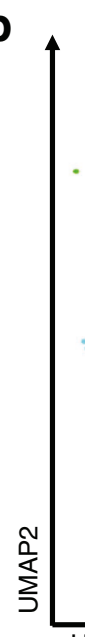

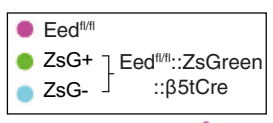

$\left.\begin{array}{c}\text { ZsG+ } \\ \text { ZsG- }\end{array}\right] \begin{gathered}\text { Eed }^{\mathrm{fuft}}:: Z \mathrm{ZsG} \text { reen } \\ :: \beta 5 \text { tCre }\end{gathered}$

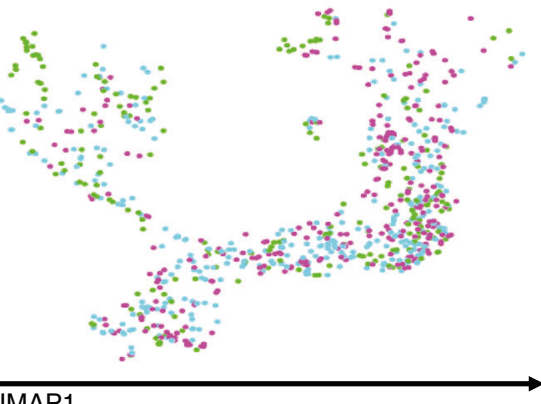

UMAP1

C
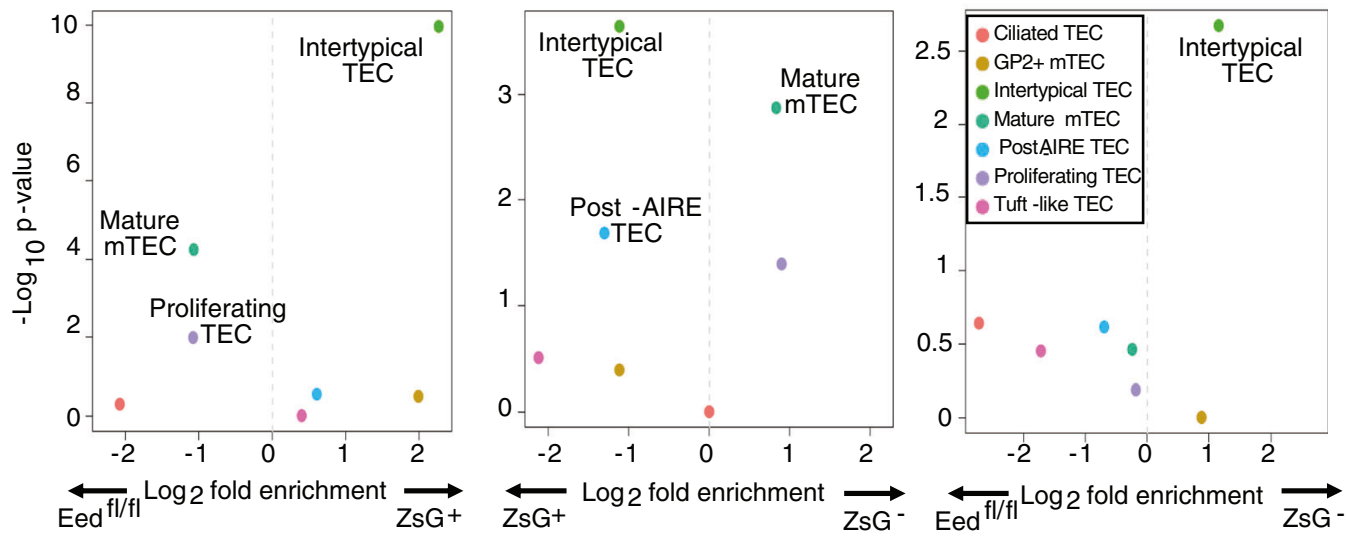

d
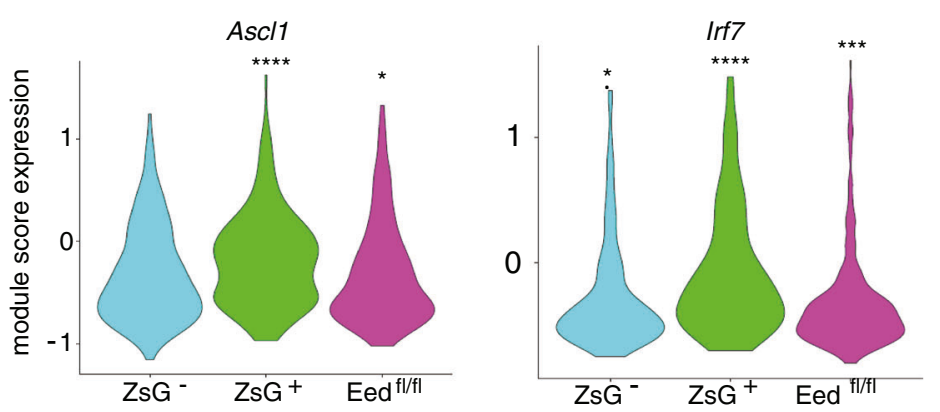

\section{* $\quad$ FDR $<0.05$ \\ ** $\quad F D R<0.01$ \\ $* * * \quad F D R<0.001$ \\ $* \star \star \star * \quad F D R<0.0001$}

Wilcoxon rank sum test

vs. all others

e

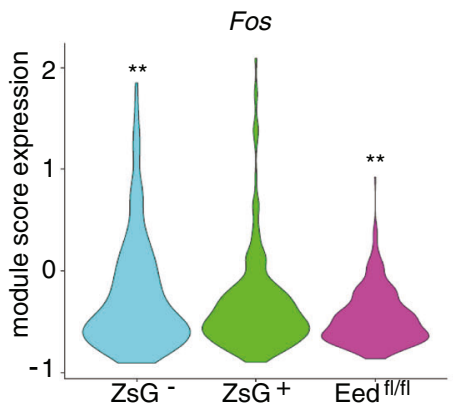

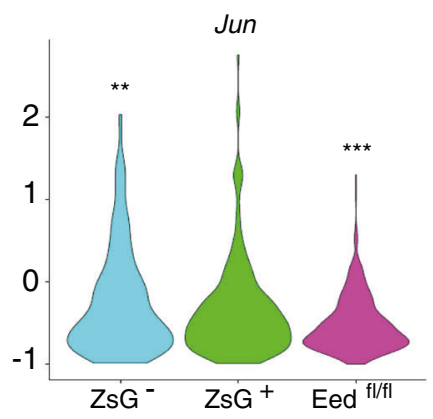

merely correlative but that loss of PRC2 activity compromises mTEC progression to a mature phenotype, through the differential induction of transcription factor networks controlled by Irf7 and Ascl1. Previous research had suggested that AIRE-regulated loci were marked by repressive chromatin modification $s^{20,49}$. However, the relatively mild reduction in the breadth of TRA expression in
Eed $d^{\mathrm{fl} / \mathrm{fl}:: \beta 5 t C r e}$ mTECs suggests that $\mathrm{H} 3 \mathrm{~K} 27 \mathrm{me} 3$ is not absolutely required for AIRE-regulated transcription. Rather, the role of PRC2 activity in TEC differentiation and suppression of alternative differentiation pathways raises the prospect that therapeutic manipulation of the TEC epigenome may be able to modulate negative selection in autoimmune diseases ${ }^{50}$. 
Fig. 9 Single-cell annotation of $\mathbf{m T E C}$ lineages. a A UMAP plot showing all datasets projected together. mTEC cell type annotation was inferred from the combination of the two reference datasets. $\mathbf{b}$ The same UMAP projection showing only single-cell RNA-seq data on $\mathrm{mTECs}$ from this study. Eed ${ }^{f l / f l}$

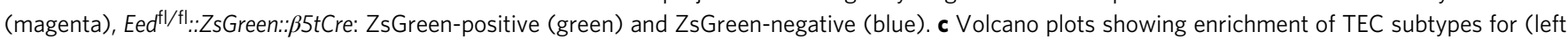

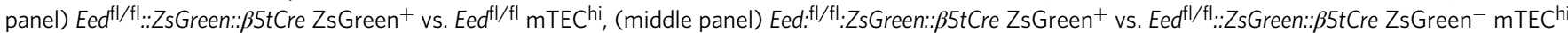

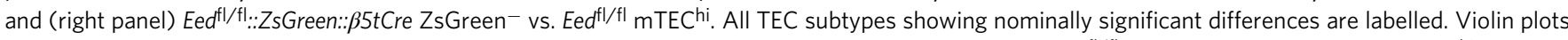
showing transcription factor network expression for transcription factor networks upregulated in (d) Eed:fl/fl:ZsGreen:: $\beta 5 t C r e ~ Z s G r e e n+m T E C h i$, and (e)

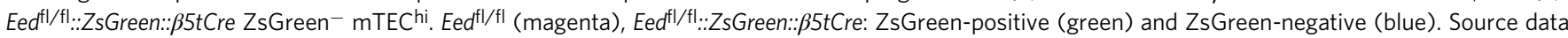
including exact statistical test values are provided in the Source Data file.

\begin{abstract}
Methods
Mice. C57BL/6 mice were obtained from Janvier. The generation of $\beta 5 \mathrm{t}-\mathrm{Cre}$, Eed, Ezh1 and Ezh2 conditionally targeted animals and TcR $\beta$ transgenic mice (YAe62 $\beta$ ) have previously been reported $25,26,32,51$. Rosa26 knock-in mice that were engineered to contain the CAG-loxP-stop-loxP-ZsGreen sequence have been described elsewhere ${ }^{52}$. Experiments used animals with both sexes until 4 weeks of age, the gender is indicated for experiments with mice older than 4 weeks. Neonatal female Ly5.2 recipients of haematopoietic stem cells were conditioned with $200 \mathrm{cGy}$ prior to receiving T cell-depleted Ly5.1 bone marrow cells $\left(3 \times 10^{6}\right)$ from TCR $\beta$ transgenic mice $(\text { YAe62 })^{32}$. Chimeric mice were analysed 4 weeks after engraftment. Rag2 $2^{-I-}$ mice were bred and maintained in our institute's SPF facility. Experimental (Cre-positive) and control (Cre-negative) mice were co-housed, C57BL/6 and $\beta 5 t-C r e$ controls were bred separately. Mice were cared for and experiments were carried out in accordance with permissions and regulations of the Cantonal Veterinary Office of Basel-Stadt (licence 2737). All animals were kept under specific pathogen-free conditions at $22 \pm 2{ }^{\circ} \mathrm{C}, 60 \pm 15 \%$ humidity, and a $12 \mathrm{~h}$ light cycle with light phase beginning at 6 a.m. with a 30 min sunrise and ending at $6 \mathrm{p}$. $\mathrm{m}$. with a $30 \mathrm{~min}$ sunset, with food and water ad libitum. Mice were euthanized by $\mathrm{CO}_{2}$ inhalation and decapitation.
\end{abstract}

Thymic epithelial cell isolation. Thymic lobes were cleaned off the fatty tissues and incubated with Liberase and DNaseI (Roche Diagnostics; 200 and $30 \mu \mathrm{g} / \mathrm{ml}$, respectively; $45 \mathrm{~min}, 37^{\circ} \mathrm{C}$ ) to obtain a cell suspension, which was subsequently filtered through a nylon mesh $(100 \mu \mathrm{m}$ pore size, Sefar Nitex $)$ to remove debris. With the exception of Fig. 1e, wild-type TEC were magnetically enriched using the AutoMACS Pro Separator (Miltenyi Biotec) to obtain sufficient cells for analysis.

Thymic macrophage and dendritic cell isolation. Cleaned thymic lobes were incubated with Collagenase D and DNaseI (Roche Diagnostics; 1 and $30 \mu \mathrm{g} / \mathrm{ml}$, respectively; $45 \mathrm{~min}, 37^{\circ} \mathrm{C}$ ) to obtain a cell suspension, which was subsequently filtered through a nylon mesh ( $100 \mu \mathrm{m}$ pore size, Sefar Nitex) to remove debris and stained.

In vivo $\mathbf{T}$ cell depletion. In vivo $\mathrm{T}$ cell depletion was achieved by i.v. injection of a cocktail of CD4 (clone GK1.5; $200 \mu \mathrm{g})$, CD8 (53-67; $100 \mu \mathrm{g}$ ) and anti-Thy1.1 (T24; $50 \mu \mathrm{g}) \mathrm{mAb}$

Flow cytometry. Thymocytes and TEC were stained as indicated, using antibodies and reagents listed in Supplementary Table 1, including dilutions. For intracellular staining, cells were fixed, permeabilized (Cytofix/Cytoperm Kit, BD Biosciences) and labelled except for Foxp3 staining, where the eBioscience transcription factor staining buffer set (ThermoFisher Scientific) was used. Stained samples were acquired on a FACSAria II or BD LSRFortessa flow cytometer with FACSDiva (BD Biosciences v8.01) and the data were analysed using the FlowJo (Treestar Inc. v9.8.3 and v10.7.1) software.

In vivo cell proliferation assay. TEC proliferation was quantified by BrdU incorporation. In short, mice were i.p. injected with $1 \mathrm{mg}$ BrdU (BD Pharmingen) diluted in $250 \mu \mathrm{l}$ sterile PBS and analysed $16 \mathrm{~h}$ later by flow cytometry following the BrdU Flow Kit protocol (BD Biosciences).

Histology. Frozen sections $(8 \mu \mathrm{m})$ of OCT embedded thymus tissue were fixed in acetone and stained either with haematoxylin and eosin or with Abs as indicated (see Supplementary Table 1). For the detection of ZsGreen, samples were first fixed in $4 \%$ paraformaldehyde prior to embedding. The tissue was sectioned using the cryostat and attached to super-frost glass slides (ThermoFisher Scientific). Sections were then stained with primary antibodies $(2 \mathrm{~h}$, room temperature), washed and developed using secondary antibodies ( $1 \mathrm{~h}$, room temperature). Images were acquired using a Zeiss LSM510 (Carl Zeiss).

In vitro T cell proliferation assay. $\mathrm{T}$ cell subpopulations were labelled for $10 \mathrm{~min}$ at room temperature with CFSE $(2.5 \mathrm{nM})$ and subsequently co-cultured for $72 \mathrm{~h}$ with irradiated (3000 cGy) Rag2 ${ }^{-l-}$ splenocytes and $1 \mu \mathrm{g} / \mathrm{ml}$ of CD3 mAb. The proliferative response was evaluated by flow cytometry and assessed by the serial dilution of CFSE label.

T cell suppression assay. Sorted naive WT lymph node CD44lo CD25neg CD4 $\mathrm{T}$ cells $\left(50,000 /\right.$ well) were cultured together with irradiated (3000 cGy) Rag2 ${ }^{-1-}$ splenic accessory cells $(100,000 /$ well $)$ and the indicated amount of sorted lymph node $\mathrm{CD} 4$ pos CD25pos $\mathrm{T}_{\text {reg }}$ in the presence of soluble CD3 $\mathrm{mAb}(0.5 \mu \mathrm{g} / \mathrm{ml})$. After $48 \mathrm{~h}, 1 \mu \mathrm{Ci}{ }^{3} \mathrm{H}$ thymidine was added overnight and cells were harvested the following day.

ELISA assay. The ELISA-Ready-Set-Go kit was used according to manufacturer's protocol to determine the concentration of IL-2. The absorbance values of each well were then acquired using an absorbance reader (Biochrom) at $450 \mathrm{~nm}$.

RNA-seq and ChIP-seq. TECs were enriched as detailed above and then sorted using a FACSAria II (BD Bioscience). RNA was isolated using RNeasy kit (Qiagen) and subjected to sequencing (TrueSeq, BGI). Sequencing data are available through the Gene Expression Omnibus (GSE112050).

Adapter sequences were trimmed using Trimmomatic ${ }^{53}$. Reads were aligned against the Ensembl transcriptome and genome (GRCm38 plus ERCC spike-ins) using HISAT (version 0.1.6) ${ }^{54}$. Reads were allocated to protein-coding gene metafeatures using HTSeq (intersection non-empty) ${ }^{55}$. Differential expression analysis on genes with mean expression at least 1 FPKM was conducted using general linear modelling in edgeR, using TMM normalization ${ }^{56}$. Genes were identified as differentially expressed using the default edgeR threshold (FDR $<0.05$ ). Clustering was performed using principal component analysis. Gene ontology enrichment was conducted in gProfiler with enrichment values estimated from 1000 permutations ${ }^{57}$.

Single-cell sequencing data were pre-processed and aligned in the same manner. Low quality cells were identified by robust PCA outlier identification on several quality control metrics (alignment proportion, ERCC spike-in proportion, number of detectable genes, proportion of reads mapping to protein-coding genes, proportion of mitochondrial transcripts, proportion of ribosomal transcripts, 3 '-to-5' coverage bias, transcriptomic variance, cell-to-mean correlation, the proportion of the library accounted for by the top 500 transcripts and GC content ${ }^{58}$. Counts were adjusted between plates using ERCC spike-ins and then by library size using SCnorm ${ }^{59}$. Cells were clustered using the tSNE component of the linnorm package, specifying pre-normalized data ${ }^{60} . \mathrm{scDD}$ was used for differential analysis of single-cell RNA-seq data ${ }^{61}$. Richness of TRA expression was assessed by resampling the increasing number of single mTECs and calculating the mean number of genes expressed in different categories of genes. Each number of cells was resampled 100 times. Statistical significance was estimated using Wilcoxon rank sum tests and correcting for multiple hypothesis testing using the Benjamini-Hochberg method. Comparison to reference singlecell RNA-seq datasets was undertaken in Seurat, integrating the reference datasets together and then integrating the single-cell RNA-seq data from this manuscript onto that ${ }^{36,37,62}$. GENIE3 and RcisTarget were used to generate transcription factor gene-regulatory networks ${ }^{63}$. Module expression for genesets was calculated using Seurat.

ChIP-seq data were processed as previously described ${ }^{64}$. Briefly reads were prealigned using BWA (version 0.5.9) then high-quality alignments were processed using Stampy. Only reads with a MAPQ score of at least 10 were taken forward for further analysis. $\mathrm{H} 3 \mathrm{~K} 27 \mathrm{me} 3$ peaks were called using irreproducibility discovery rate analysis (specifying broad peaks). Significance of ChIP/input changes between samples were estimated using Wilcoxon rank sum tests and correcting for multiple hypothesis testing using the Benjamini-Hochberg method.

RNA-seq and ChIP-seq data are available at the Gene Expression Omnibus (GSE112050 and GSE114713). AIRE ChIP-seq data were downloaded from GSE9259765

T cell sorting for TCR repertoire analysis. Whole-thymus suspensions were prepared by pushing organs through a $70 \mu \mathrm{m}$ sieve into PBS containing $2 \% \mathrm{v} / \mathrm{v}$ heat-inactivated fetal calf serum and $2 \mathrm{mM}$ EDTA (designated sort buffer). For CCR7 staining of thymocytes, suspensions were incubated for $30 \mathrm{~min}$ at $37^{\circ} \mathrm{C}$ in pre-warmed sort buffer containing anti-CCR7. Cells were then pelleted by 
a
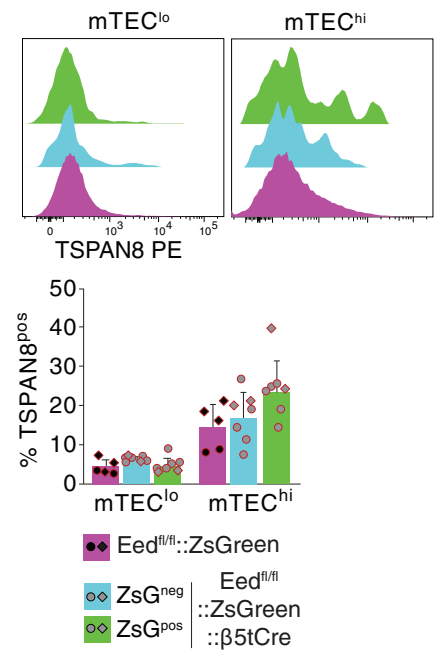

C

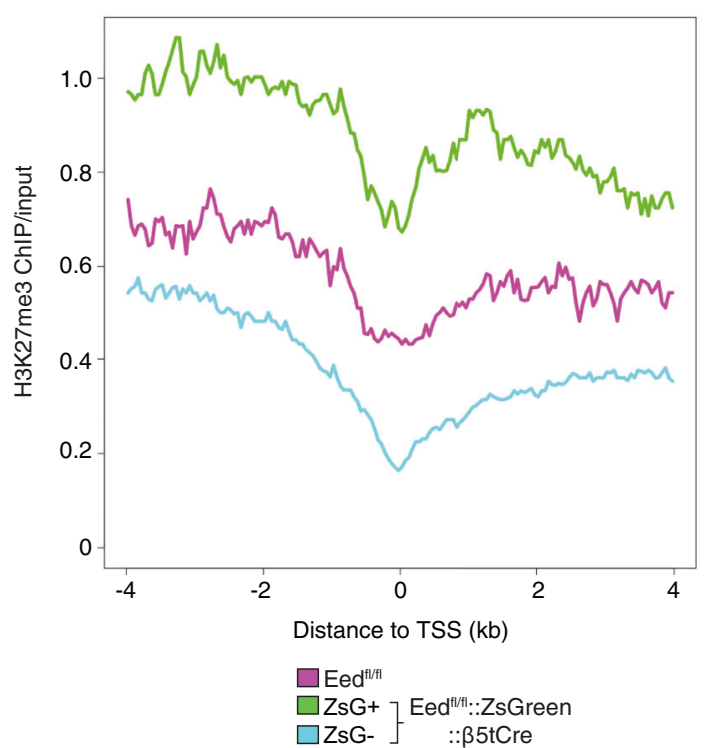

b
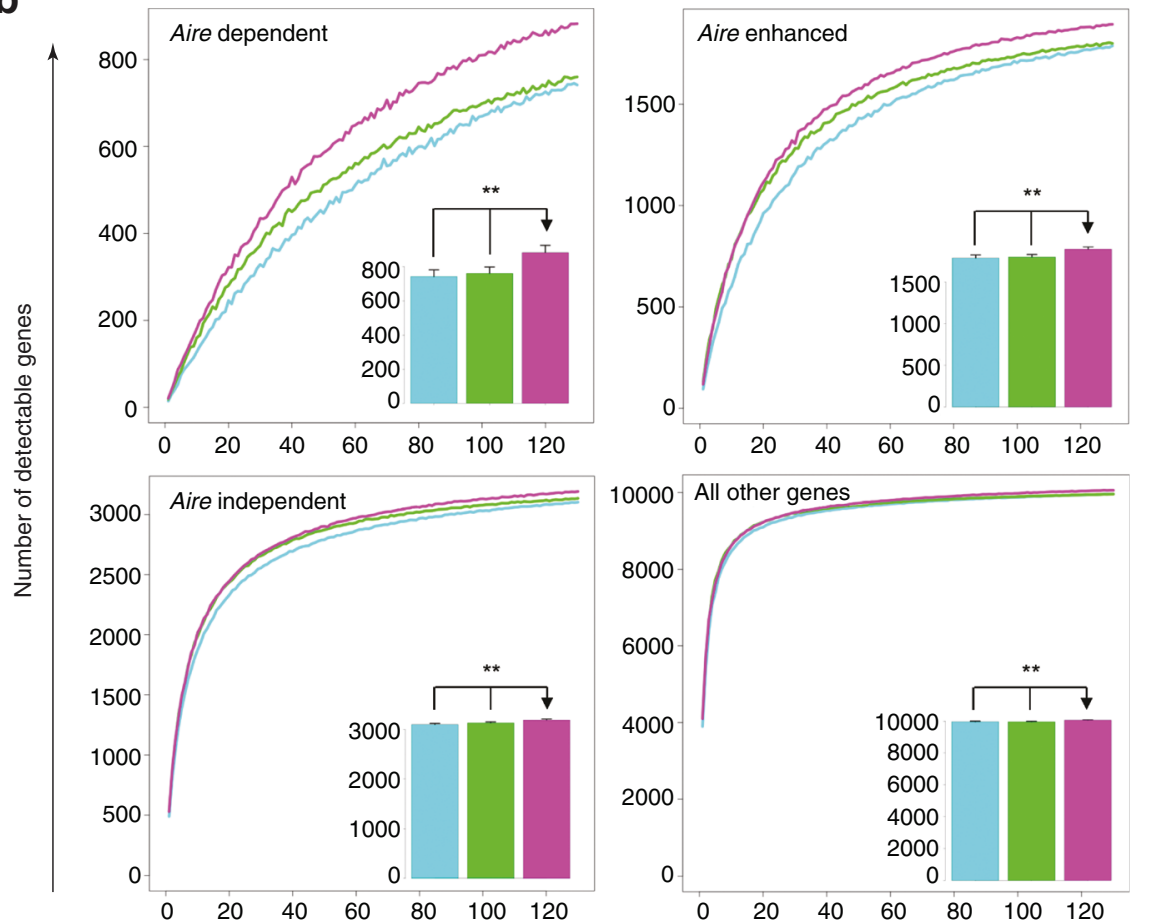

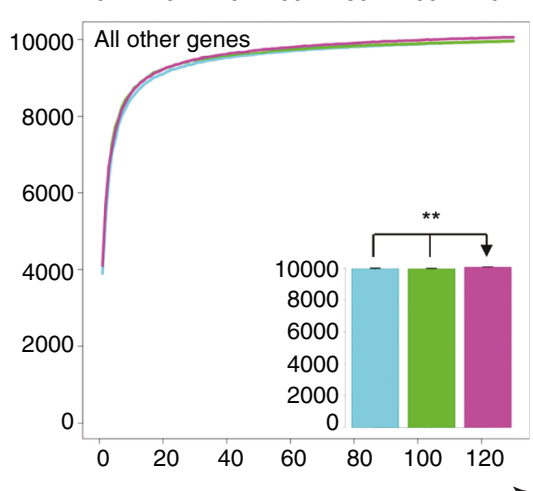

Number of cells

Fig. 10 The effects of Eed loss on AIRE-dependent gene expression and H3K27me3 repressed genes. a Representative histograms and quantitative analysis of TSPAN8 expression in mTEClo and mTEChi. Graph shows mean with SD, data are pooled from two independent experiments identified by

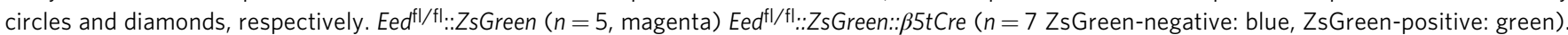
$n=$ biologically independent replicates per group, mice were 3-4 weeks of age. $\mathbf{b}$ The number of detectable genes from randomly sampled single ZsGreen-negative (blue), ZsGreen-positive (green) and Eedfl/fl (purple) mTEChi. The inset bar graphs show the mean number of detectable genes when resampling 130 single $\mathrm{mTEC}$ hi. Error bars indicate the standard deviation. ${ }^{\star *}$ Corrected $p<0.001$ (two-sided Wilcoxon rank sum test with Benjamini-Hochberg correction). Prior to analysis, cells were downsampled to 200,000 transcriptomic fragments. c Median wild-type H3K27me3 ChIP/input in $50 \mathrm{bp}$ bins around the transcription start site (TSS) for genes significantly upregulated in mTEChi. Genes were those most highly expressed in ZsGreen-positive (green), ZsGreen-negative (blue) or Eed ${ }^{f l / f l}$ (magenta). Comparisons between ZsGreen-positive vs. ZsGreen-negative

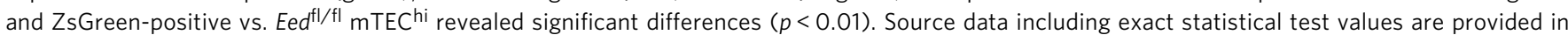
the Source Data file. 
centrifugation and incubated for $30 \mathrm{~min}$ in sort buffer at $4{ }^{\circ} \mathrm{C}$ containing assortments of fluorochrome-conjugated monoclonal antibodies. After washing, cells were passed through a $40 \mu \mathrm{m}$ sieve before sorting into $1.5 \mathrm{ml}$ Eppendorf tubes containing $350 \mu \mathrm{l}$ Qiagen Buffer RLT, which were frozen by pushing into dry ice and then stored at $-80^{\circ} \mathrm{C}$ until RNA isolation. Cell source, type and number are displayed in Supplementary Table 2.

TCR sequence acquisition in YAe62-tg T cells. RNA was isolated using Qiagen RNeasy Mini kits with an elution volume of $22 \mu \mathrm{l}$, from which $12 \mu \mathrm{l}$ were used to synthesize cDNA (Quantitect RT, QIAgen). Using $5 \mu$ lof cDNA template per reaction, TCRa transcripts were PCR amplified using a mix of 24 Trav-specific forward primers and a single Trac-specific reverse primer (Supplementary Table 3). Forward and reverse primers had distinct $5^{\prime}$ overhang adapter sequences that enabled addition of sample-specific indices and P5/P7 sequencing adapters in a second PCR using the Illumina Nextera XT DNA library preparation kit. Before the second PCR, magnetic beads (Agencourt AMPure XP, Beckman Coulter) were used to enrich amplicons $>100 \mathrm{bp}$. Conditions for the first PCR were $98^{\circ} \mathrm{C}$ for 5 min, 20 cycles of $98^{\circ} \mathrm{C}$ for $10 \mathrm{~s}, 60^{\circ} \mathrm{C}$ for $30 \mathrm{~s}$ and $72^{\circ} \mathrm{C}$ for $30 \mathrm{~s}$, followed by $72{ }^{\circ} \mathrm{C}$ for $2 \mathrm{~min}$ and for the second PCR were $72^{\circ} \mathrm{C}$ for $3 \mathrm{~min}$ and $98^{\circ} \mathrm{C}$ for $30 \mathrm{~s}, 20$ cycles of $98^{\circ} \mathrm{C}$ for $10 \mathrm{~s}, 63^{\circ} \mathrm{C}$ for $30 \mathrm{~s}$ and $72{ }^{\circ} \mathrm{C}$ for $30 \mathrm{~s}$, followed by $72^{\circ} \mathrm{C}$ for 1 min. After determining amplicon concentrations using a QIAxcel capillary electrophoresis machine (Qiagen), equimolar amounts of amplicons from 46 samples were pooled into a single tube, concentrated using magnetic beads (Agencourt AMPure XP, Beckman Coulter) and then 300-500 bp amplicons were gel-purified before 250 bp paired-end sequencing on an Illumina MiSeq machine. The full TCR sequence dataset is available at the Sequence Read Archive (BioProject ID PRJNA565651) and processed data are available from the authors on request.

TCR sequence processing. Sequences were aligned to Trav and Traj gene segments, and CDR3 amino acid sequences were ascertained, using molecular identifier groups-based error correction (MIGEC) software ${ }^{66}$. Subsequent analyses were performed using RStudio software. All data were filtered to exclude non-functional CDR3 sequences that were either out-of-frame or contained a stop codon. To avoid overestimating TCR diversity due to PCR or sequencing errors, sequences detected fewer than 3 times in any given sample were excluded from further analysis. Sequences that aligned with $>1$ Trav paralog were assumed to use the Trav paralog listed first by MIGEC except for Supplementary Fig. 5d, e, from which these ambiguous sequences were excluded. A unique combination of Trav segment and CDR3 amino acid sequence was defined as a clonotype. Each clonotype was counted only once per sample. All clonotypes detected in samples of a given combination of genotype and T cell subset were pooled to form a TCR catalogue. Unless otherwise indicated, analyses were performed at the TCR catalogue level. Self-reactive CDR3 doublet expression at positions 6 and 7 of the CDR3 was determined by calculating the proportion of sequences using any of the 175 amino acid doublets identified as promoting self-reactivity when present at this position. For this analysis only, position 1 of the CDR3 was designated as the conserved cysteine at the CDR3 N-terminus. For diversity analyses, the iNEXT software package was used to plot coverage-based rarefaction and extrapolation curves, plus relative diversity measurements, following the approach of Chao and Jost ${ }^{67}$ whose term "individual" we equate with "clone", and "species" we equate with "clonotype". Catalogues were assembled in a clone-based (individual-based) abundance format and clonotype (species) diversity estimates were made using the diversity order (Hill number or $q$ ). Morisita Horn indices were computed using EstimateS (Version 9.1.0, R. K. Colwell, http://purl.oclc.org/estimates) with clonotype abundance equal to the number of mice in which the clonotype was detected in a given TCR catalogue.

TCR $\alpha$ chain analysis in non-transgenic $\mathbf{T}$ cells. TCR $\alpha$ repertoire analyses were performed by SMARTer 5'RACE cDNA amplification on thymocytes as previously described $^{68}$. CDR3 sequences were identified using LymAnalyzer ${ }^{69}$. Each unique TRAV-TRAJ clonotype was recorded in a FlowJo-based file to calculate frequencies of TRAV and TRAJ proximal and distal gene usage. Principal component analysis and hierarchical clustering (HC) were performed using FactomineR (http://factominer.free.fr/) and Factoextra (http://www.sthda.com/english/rpkgs/ factoextra). The following 8 parameters were extracted from TCR $\alpha$ repertoire analysis for HC determination: relative frequencies of distal and proximal TRAV segments and frequencies of distal, medium, and distal TRAJ elements within proximal and distal TRAV containing transcripts.

Statistical analyses. Statistical analyses for data presented were performed using Student's $t$ test (unpaired, two-tailed). $p<0.05$ was considered significant. The statistical evaluation of RNA-seq data is described in separate statistical analysis section above. The sample size used and estimates of variation within groups were based on published results using similar approaches. No randomization was done for animal studies and investigators were not blinded to experimental group allocations.
Reporting summary. Further information on research design is available in the Nature Research Reporting Summary linked to this article.

\section{Data availability}

The full TCR sequence dataset is available at the Sequence Read Archive (BioProject ID PRJNA565651) and processed data are available from the authors on request. RNA-seq and ChIP-seq data are available at the Gene Expression Omnibus (GSE112050 and GSE114713). AIRE ChIP-seq data were downloaded from GSE92597. Other data that support the findings of this study are available from the corresponding author upon reasonable request. Source data are provided with this paper.

Received: 11 June 2020; Accepted: 31 May 2021; Published online: 24 June 2021

\section{References}

1. Abramson, J. \& Anderson, G. Thymic epithelial cells. Annu. Rev. Immunol. 35, 85-118 (2017).

2. Rossi, S. W., Jenkinson, W. E., Anderson, G. \& Jenkinson, E. J. Clonal analysis reveals a common progenitor for thymic cortical and medullary epithelium. Nature 441, 988-991 (2006).

3. Bleul, C. C. et al. Formation of a functional thymus initiated by a postnatal epithelial progenitor cell. Nature 441, 992-996 (2006).

4. Ribeiro, A. R., Rodrigues, P. M., Meireles, C., Di Santo, J. P. \& Alves, N. L. Thymocyte selection regulates the homeostasis of IL-7-expressing thymic cortical epithelial cells in vivo. J. Immunol. 191, 1200-1209 (2013).

5. Baik, S., Jenkinson, E. J., Lane, P. J., Anderson, G. \& Jenkinson, W. E. Generation of both cortical and Aire $(+)$ medullary thymic epithelial compartments from CD205(+) progenitors. Eur. J. Immunol. 43, 589-594 (2013).

6. Ohigashi, I. et al. Adult thymic medullary epithelium is maintained and regenerated by lineage-restricted cells rather than bipotent progenitors. Cell Rep. 13, 1432-1443 (2015).

7. Mayer, C. E. et al. Dynamic spatio-temporal contribution of single $\beta 5 t+$ cortical epithelial precursors to the thymus medulla. Eur. J. Immunol. 46, 846-856 (2016).

8. Sansom, S. N. et al. Population and single-cell genomics reveal the Aire dependency, relief from polycomb silencing, and distribution of self-antigen expression in thymic epithelia. Genome Res. 24, 1918-1931 (2014).

9. Bonasio, R. et al. Clonal deletion of thymocytes by circulating dendritic cells homing to the thymus. Nat. Immunol. 7, 1092-1100 (2006).

10. Hadeiba, H. et al. Plasmacytoid dendritic cells transport peripheral antigens to the thymus to promote central tolerance. Immunity 36, 438-450 (2012).

11. Yamano, T. et al. Thymic B cells are licensed to present self antigens for central T cell tolerance induction. Immunity 42, 1048-1061 (2015).

12. Anderson, M. S. \& Su, M. A. AIRE expands: new roles in immune tolerance and beyond. Nat. Rev. Immunol. 16, 247-258 (2016).

13. Villasenor, J., Besse, W., Benoist, C. \& Mathis, D. Ectopic expression of peripheral-tissue antigens in the thymic epithelium: probabilistic, monoallelic, misinitiated. Proc. Natl Acad. Sci. USA 105, 15854-15859 (2008).

14. Derbinski, J., Pinto, S., Rosch, S., Hexel, K. \& Kyewski, B. Promiscuous gene expression patterns in single medullary thymic epithelial cells argue for a stochastic mechanism. Proc. Natl Acad. Sci. USA 105, 657-662 (2008).

15. Meredith, M., Zemmour, D., Mathis, D. \& Benoist, C. Aire controls gene expression in the thymic epithelium with ordered stochasticity. Nat. Immunol. 16, 942-949 (2015)

16. Pinto, S. et al. Overlapping gene coexpression patterns in human medullary thymic epithelial cells generate self-antigen diversity. Proc. Natl Acad. Sci. USA 110, E3497-E3505 (2013).

17. Rattay, K., Meyer, H. V., Herrmann, C., Brors, B. \& Kyewski, B. Evolutionary conserved gene co-expression drives generation of self-antigen diversity in medullary thymic epithelial cells.J. Autoimmun. 67, 65-75 (2016).

18. Brennecke, P. et al. Single-cell transcriptome analysis reveals coordinated ectopic gene-expression patterns in medullary thymic epithelial cells. Nat. Immunol. 16, 933-941 (2015).

19. Miragaia, R. J. et al. Single-cell RNA-sequencing resolves self-antigen expression during mTEC development. Sci. Rep. 8, 685 (2018).

20. Koh, A. S. et al. Rapid chromatin repression by Aire provides precise control of immune tolerance. Nat. Immunol. 19, 162-172 (2018).

21. Smits, A. H., Jansen, P. W., Poser, I., Hyman, A. A. \& Vermeulen, M. Stoichiometry of chromatin-associated protein complexes revealed by labelfree quantitative mass spectrometry-based proteomics. Nucleic Acids Res. 41, e28 (2013).

22. Kasinath, V. et al. Structures of human PRC2 with its cofactors AEBP2 and JARID2. Science 359, 940-944 (2018). 
23. Hu, D. et al. The Mll2 branch of the COMPASS family regulates bivalent promoters in mouse embryonic stem cells. Nat. Struct. Mol. Biol. 20, 1093-1097 (2013).

24. Faust, C., Schumacher, A., Holdener, B. \& Magnuson, T. The eed mutation disrupts anterior mesoderm production in mice. Development 121, 273-285 (1995).

25. Xie, H. et al. Polycomb repressive complex 2 regulates normal hematopoietic stem cell function in a developmental-stage-specific manner. Cell Stem Cell 14, 68-80 (2014)

26. Ohigashi, I. et al. Aire-expressing thymic medullary epithelial cells originate from 35 t-expressing progenitor cells. Proc. Natl Acad. Sci. USA 110, 9885-9890 (2013).

27. Daley, S. R., Hu, D. Y. \& Goodnow, C. C. Helios marks strongly autoreactive $\mathrm{CD} 4+\mathrm{T}$ cells in two major waves of thymic deletion distinguished by induction of PD-1 or NF-kB. J. Exp. Med. 210, 269-285 (2013).

28. Azzam, H. S. et al. Fine tuning of TCR signaling by CD5. J. Immunol. 166, 5464-5472 (2001).

29. Scott, R. S. et al. Phagocytosis and clearance of apoptotic cells is mediated by MER. Nature 411, 207-211 (2001).

30. Shrimpton, R. E. et al. CD205 (DEC-205): a recognition receptor for apoptotic and necrotic self. Mol. Immunol. 46, 1229-1239 (2009).

31. Proietto, A. I. et al. Dendritic cells in the thymus contribute to T-regulatory cell induction. Proc. Natl Acad. Sci. USA 105, 19869-19874 (2008).

32. Stadinski, B. D. et al. A role for differential variable gene pairing in creating $T$ cell receptors specific for unique major histocompatibility ligands. Immunity 35, 694-704 (2011).

33. Stadinski, B. D. et al. Hydrophobic CDR3 residues promote the development of self-reactive T cells. Nat. Immunol. 17, 946-955 (2016).

34. Daley, S. R. et al. Cysteine and hydrophobic residues in CDR3 serve as distinct T-cell self-reactivity indices. J. Allergy Clin. Immunol. 144, 333-336 (2019).

35. Wirasinha, R. C. et al. $\alpha \beta$ T-cell receptors with a central CDR3 cysteine are enriched in CD8aa intraepithelial lymphocytes and their thymic precursors. Immunol. Cell Biol. 96, 553-561 (2018).

36. Baran-Gale, J. et al. Ageing compromises mouse thymus function and remodels epithelial cell differentiation. eLife 9, e56221 (2020).

37. Dhalla, F. et al. Biologically indeterminate yet ordered promiscuous gene expression in single medullary thymic epithelial cells. EMBO J. 39, e101828 (2020).

38. Gordon, J. et al. Functional evidence for a single endodermal origin for the thymic epithelium. Nat. Immunol. 5, 546-553 (2004).

39. Witt, C. M., Raychaudhuri, S., Schaefer, B., Chakraborty, A. K. \& Robey, E. A. Directed migration of positively selected thymocytes visualized in real time. PLoS Biol. 3, el60 (2005)

40. Guo, J. et al. Regulation of the TCRalpha repertoire by the survival window of CD4(+)CD8(+) thymocytes. Nat. Immunol. 3, 469-476 (2002).

41. Apavaloaei, A., Rouette, A., Brochu, S. \& Perreault, C. Psmb11 molds the transcriptome of cortical TECs and CD8 thymocytes. J. Immunol. 198 , 202.214-202.214 (2017).

42. Singarapu, N. et al. Polycomb repressive complex 2 is essential for development and maintenance of a functional TEC compartment. Sci. Rep. 8 , 14335 (2018)

43. Kroger, C. J., Spidale, N. A., Wang, B. \& Tisch, R. Thymic dendritic cell subsets display distinct efficiencies and mechanisms of intercellular MHC transfer. J. Immunol. 198, 249-256 (2017).

44. Coquet, J. M. et al. Epithelial and dendritic cells in the thymic medulla promote $\mathrm{CD} 4{ }^{+} \mathrm{Foxp}^{+}{ }^{+}$regulatory $\mathrm{T}$ cell development via the $\mathrm{CD} 27-\mathrm{CD} 70$ pathway. J. Exp. Med. 210, 715-728 (2013)

45. Perry, J. S. A. et al. Distinct contributions of Aire and antigen-presenting-cell subsets to the generation of self-tolerance in the thymus. Immunity $\mathbf{4 1}$, 414-426 (2014).

46. Lancaster, J. N. et al. Live-cell imaging reveals the relative contributions of antigen-presenting cell subsets to thymic central tolerance. Nat. Commun. 10, 2220 (2019).

47. Anderson, M. S. et al. Projection of an immunological self shadow within the thymus by the aire protein. Science 298, 1395-1401 (2002).

48. Handel, A. E. et al. Comprehensively profiling the chromatin architecture of tissue restricted antigen expression in thymic epithelial cells over development. Front Immunol. 9, 2120 (2018).

49. Sansom, S.N. et al. Population and single cell genomics reveal the Airedependency, relief from polycomb silencing and distribution of self-antigen expression in thymic epithelia.Genome Res. 24, 1918-1931 (2014).

50. Kim, K. H. \& Roberts, C. W. Targeting EZH2 in cancer. Nat. Med 22, 128-134 (2016).

51. Ezhkova, E. et al. EZH1 and EZH2 cogovern histone H3K27 trimethylation and are essential for hair follicle homeostasis and wound repair. Genes Dev. 25, 485-498 (2011).

52. Madisen, L. et al. A robust and high-throughput Cre reporting and characterization system for the whole mouse brain. Nat. Neurosci. 13, 133-140 (2010).
53. Bolger, A. M., Lohse, M. \& Usadel, B. Trimmomatic: a flexible trimmer for Illumina sequence data. Bioinformatics 30, 2114-2120 (2014).

54. Kim, D., Langmead, B. \& Salzberg, S. L. HISAT: a fast spliced aligner with low memory requirements. Nat. Methods 12, 357-360 (2015).

55. Anders, S., Pyl, P. T. \& Huber, W. HTSeq-a Python framework to work with high-throughput sequencing data. Bioinformatics 31, 166-169 (2015).

56. Robinson, M. D., McCarthy, D. J. \& Smyth, G. K. edgeR: a Bioconductor package for differential expression analysis of digital gene expression data. Bioinformatics 26, 139-140 (2010).

57. Reimand, J. et al. g:Profiler-a web server for functional interpretation of gene lists (2016 update). Nucleic Acids Res. 44, W83-W89 (2016).

58. Ilicic, T. et al. Classification of low quality cells from single-cell RNA-seq data. Genome Biol. 17, 29 (2016).

59. Bacher, R. et al. SCnorm: robust normalization of single-cell RNA-seq data. Nat. Methods 14, 584-586 (2017).

60. Yip, S. H., Wang, P., Kocher, J.-P. A., Sham, P. C. \& Wang, J. Linnorm: improved statistical analysis for single cell RNA-seq expression data. Nucleic Acids Res. 45, 13097 (2017)

61. Korthauer, K. D. et al. A statistical approach for identifying differential distributions in single-cell RNA-seq experiments. Genome Biol. 17, 222 (2016).

62. Stuart, T. et al. Comprehensive integration of single-cell data. Cell 177, 1888-1902.e1821 (2019).

63. Aibar, S. et al. SCENIC: single-cell regulatory network inference and clustering. Nat. Methods 14, 1083-1086 (2017).

64. Žuklys, S. et al. Foxn1 regulates key target genes essential for $\mathrm{T}$ cell development in postnatal thymic epithelial cells. Nat. Immunol. 17, 1206-1215 (2016)

65. Bansal, K., Yoshida, H., Benoist, C. \& Mathis, D. The transcriptional regulator Aire binds to and activates super-enhancers. Nat. Immunol. 18, 263-273 (2017).

66. Shugay, M. et al. Towards error-free profiling of immune repertoires. Nat. Methods 11, 653-655 (2014).

67. Chao, A. \& Jost, L. Coverage-based rarefaction and extrapolation: standardizing samples by completeness rather than size. Ecology 93, 25332547 (2012)

68. Vera, G. et al. Cernunnos deficiency reduces thymocyte life span and alters the T cell repertoire in mice and humans. Mol. Cell Biol. 33, 701-711 (2013).

69. Yu, Y., Ceredig, R. \& Seoighe, C. LymAnalyzer: a tool for comprehensive analysis of next generation sequencing data of $\mathrm{T}$ cell receptors and immunoglobulins. Nucleic Acids Res. 44, e31 (2016).

\section{Acknowledgements}

We thank E. Christen, E. Terszowska and A. Offinger for excellent technical support. E. Ezhkova for providing Ezh mouse strains, M. Gaio and S. Harris for secretarial assistance, the Computational Biology Research Group at the Weatherall Institute of Molecular Medicine for server support and K. Weinacht (Stanford) for logistics. This work was supported by grants from the Swiss National Foundation (3100- 68310.02 and $3100-122558$ to G.A.H), The Wellcome Trust (105045/Z/14/Z to G.A.H.; 100643/Z/12/Z to A.E.H.), the NHMRC of Australia (1107464 to S.R.D.) and the National Institutes of Health Research (Clinical Lectureship to A.E.H.)

\section{Author contributions}

T. B., A.E.H., S.R.D., H.Y.T., J.dV. and G.A.H. designed the experiments, T.B. A.E.H. H.Y.T., R.C.W., S.R.D., K.H., S.Z., B.R. and J.dV. performed the experiments. T.B., A.E.H., H.Y.T., S.R.D., J.dV. and G.A.H. analysed and/or interpreted results. G.A.H., A.E.H., S.R.D, S.H.O. and T.B. wrote the manuscript.

\section{Competing interests}

The authors declare no competing interests.

\section{Additional information}

Supplementary information The online version contains supplementary material available at https://doi.org/10.1038/s41467-021-24158-w.

Correspondence and requests for materials should be addressed to G.A.H.

Peer review information Nature Communications thanks Panagiotis Ntziachristos and the other, anonymous, reviewer(s) for their contribution to the peer review of this work.

Reprints and permission information is available at http://www.nature.com/reprints

Publisher's note Springer Nature remains neutral with regard to jurisdictional claims in published maps and institutional affiliations. 
(c) (i) Open Access This article is licensed under a Creative Commons Attribution 4.0 International License, which permits use, sharing, adaptation, distribution and reproduction in any medium or format, as long as you give appropriate credit to the original author(s) and the source, provide a link to the Creative Commons license, and indicate if changes were made. The images or other third party material in this article are included in the article's Creative Commons license, unless indicated otherwise in a credit line to the material. If material is not included in the article's Creative Commons license and your intended use is not permitted by statutory regulation or exceeds the permitted use, you will need to obtain permission directly from the copyright holder. To view a copy of this license, visit http://creativecommons.org/ licenses/by/4.0/.

(C) The Author(s) 2021 